\title{
iPTF 16asu: A Luminous, Rapidly Evolving, and High-velocity Supernova
}

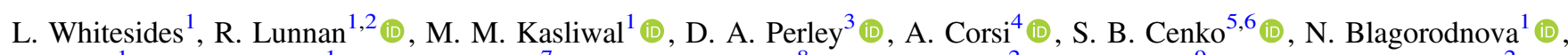

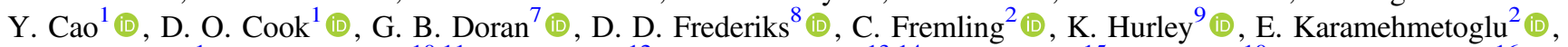

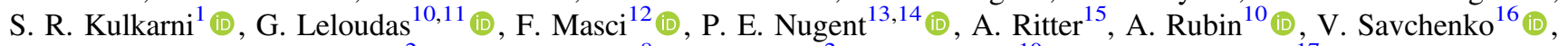 \\ J. Sollerman ${ }^{2}$ (1), D. S. Svinkin ${ }^{8}$ (1) , F. Taddia ${ }^{2}$, P. Vreeswijk ${ }^{10}$ (1) , and P. Wozniak ${ }^{17}$ \\ ${ }^{1}$ Department of Astronomy, California Institute of Technology, 1200 East California Boulevard, Pasadena, CA 91125, USA; 1whitesides@caltech.edu \\ ${ }^{2}$ The Oskar Klein Centre \& Department of Astronomy, Stockholm University, AlbaNova, SE-106 91 Stockholm, Sweden \\ ${ }^{3}$ Astrophysics Research Institute, Liverpool John Moores University, IC2, Liverpool Science Park, 146 Browlow Hill, Liverpool L3 5RF, UK \\ ${ }^{4}$ Department of Physics and Astronomy, Texas Tech University, Box 41051, Lubbock, TX 79409-1051, USA \\ ${ }^{5}$ Astrophysics Science Division, NASA Goddard Space Flight Center, Mail Code 661, Greenbelt, MD 20771, USA \\ ${ }^{6}$ Joint Space-Science Institute, University of Maryland, College Park, MD 20742, USA \\ 7 Jet Propulsion Laboratory, California Institute of Technology, Pasadena, CA 91109, USA \\ ${ }^{8}$ Ioffe Institute, Politekhnicheskaya 26, St. Petersburg 194021, Russia \\ ${ }^{9}$ University of California, Berkeley, Space Sciences Laboratory, 7 Gauss Way, Berkeley, CA 94720-7450, USA \\ ${ }^{10}$ Department of Particle Physics and Astrophysics, Weizmann Institute of Science, 234 Herzl Street, Rehovot, Israel \\ ${ }^{11}$ Dark Cosmology Centre, Niels Bohr Institute, University of Copenhagen, Juliane Maries Vej 30, DK-2100 Copenhagen, Denmark \\ ${ }^{12}$ Infrared Processing and Analysis Center, California Institute of Technology, MS 100-22, Pasadena, CA 91125, USA \\ ${ }^{13}$ Lawrence Berkeley National Laboratory, 1 Cyclotron Road Berkeley, CA 94720, USA \\ ${ }^{14}$ Department of Astronomy, University of California, 501 Campbell Hall, Berkeley, CA 94720-3411, USA \\ ${ }^{15}$ Department of Astrophysical Sciences, Princeton University, Princeton, NJ 08544, USA \\ ${ }^{16}$ ISDC, Department of Astronomy, University of Geneva, Chemin d'Écogia, 16 CH-1290 Versoix, Switzerland \\ ${ }^{17}$ Los Alamos National Laboratory, MS-D466, Los Alamos, NM 87545, USA \\ Received 2017 June 14; revised 2017 October 10; accepted 2017 November 6; published 2017 December 18
}

\begin{abstract}
Wide-field surveys are discovering a growing number of rare transients whose physical origin is not yet well understood. Here we present optical and UV data and analysis of intermediate Palomar Transient Factory (iPTF) 16asu, a luminous, rapidly evolving, high-velocity, stripped-envelope supernova (SN). With a rest-frame rise time of just four days and a peak absolute magnitude of $M_{\mathrm{g}}=-20.4 \mathrm{mag}$, the light curve of iPTF 16asu is faster and more luminous than that of previous rapid transients. The spectra of iPTF 16asu show a featureless blue continuum near peak that develops into an SN Ic-BL spectrum on the decline. We show that while the late-time light curve could plausibly be powered by ${ }^{56} \mathrm{Ni}$ decay, the early emission requires a different energy source. Nondetections in the X-ray and radio strongly constrain the energy coupled to relativistic ejecta to be at most comparable to the class of low-luminosity gamma-ray bursts (GRBs). We suggest that the early emission may have been powered by either a rapidly spinning-down magnetar or by shock breakout in an extended envelope of a very energetic explosion. In either scenario a central engine is required, making iPTF 16asu an intriguing transition object between superluminous SNe, SNe Ic-BL, and low-luminosity GRBs.
\end{abstract}

Key words: gamma-ray burst: general - shock waves - stars: magnetars - supernovae: general - supernovae: individual (iPTF 16asu)

Supporting material: machine-readable table

\section{Introduction}

Many new and unusual astrophysical transients have been discovered recently by wide-field surveys that regularly monitor the night sky. Supernovae (SNe) are traditionally classified based on their spectra (see Filippenko 1997 for a review) and fall into two main groups: $\mathrm{SNe} \mathrm{II} / \mathrm{Ibc}$, which originate from the core collapse of massive stars, and SNe Ia, which are produced by thermonuclear disruptions of white dwarfs. The advent of dedicated wide-field surveys with increased survey speeds has led to the discovery of exotic types of $\mathrm{SNe}$ and other transient events both inside and outside of galaxies (see Kasliwal 2012 for a review). These rare detections have necessitated the establishment of new categories of $\mathrm{SNe}$ such as Ca-rich gap transients (e.g., Perets et al. 2010), .Ia explosions (e.g., Kasliwal et al. 2010), intermediate-luminosity red transients (e.g., Prieto et al. 2008), and superluminous SNe (e.g., Quimby et al. 2011), which demand different physical models than those previously used to explain SNe. The physics powering transient objects in our universe continues to be a rich topic of exploration.

This diverse landscape of transients is illustrated in Figure 1, shown in the phase space of rise time (explosion to peak) versus peak luminosity. As peak luminosities are not always available in the same filters, this figure should not be used for quantitative comparisons but rather as an illustration of the approximate areas inhabited by different transients in this phase space. SNe Ia, shown as a green diamond, act as standardizable candles (Phillips 1993). They exhibit a tight range of luminosities and rise times (Hayden et al. 2010). SNe II, shown as large cyan circles, are characterized by fast rise times but relatively low luminosities (Rubin et al. 2016). SNe Ibc, shown as small magenta circles, are more heterogeneous but tend to rise more slowly and become brighter than SNe II (Taddia et al. 2015); those with broad spectral features ( $\mathrm{SNe}$ Ic-BL), denoted as magenta diamonds, generally reach higher peak luminosities than typical SNe Ibc (Corsi et al. 2012, 2017). Superluminous supernovae (SLSNe), shown as small 


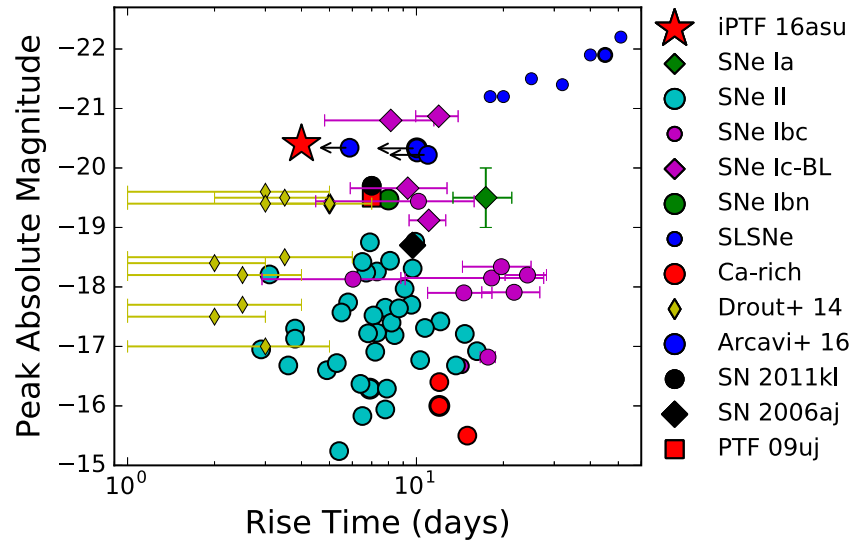

Figure 1. Rest-frame rise time (explosion to peak) vs. peak absolute magnitude of a variety of types of SNe. iPTF 16asu, shown as a red star, is unique in its combination of high luminosity and fast rise time. Data from Hayden et al. (2010) ( $B$ band), Rubin et al. (2016) ( $R$ band), Taddia et al. (2015) ( $g$ band), Hosseinzadeh et al. (2017) (template), Barbary et al. (2009) ( $i$ band), Pastorello et al. (2010) ( $B$ band), Quimby et al. (2011) ( $u$ band), Lunnan et al. (2013) ( $r$ band), Inserra et al. (2013) ( $r$ band), Chomiuk et al. (2011) ( $z$ band), Drout et al. (2014) ( $r$ band; yellow diamonds), Arcavi et al. (2016) ( $r$ band; large blue circles), Greiner et al. (2015) ( $i$ band), Lunnan et al. (2017) ( $r$ band), Kasliwal et al. (2012) ( $r$ band), and Ofek et al. (2010) (NUV band). Where possible rise times are given in the band closest to the iPTF 16asu rest-frame $g$ band.

blue circles, are extremely bright transients with very long rise times (Quimby et al. 2011; Gal-Yam 2012).

Transients that rise and decay rapidly are difficult to detect, requiring a sufficiently high cadence over a sufficiently large volume, rapid triggering, and follow-up. Improvements in these areas have enabled the discovery of objects that populate this previously empty region of short time scales at a wide range of luminosities. Drout et al. (2014) searched the Pan-STARRS1 Medium Deep Survey for rapidly evolving transients, resulting in the sample of objects shown in yellow in Figure 1. Recently, Arcavi et al. (2016) presented another four rapidly evolving objects (shown as large blue circles), with intermediate luminosities between SLSNe and the other types of known $\mathrm{SNe}$. These objects are also similar in rise time and luminosity to SN 2011kl, a unique event associated with an ultra-long gamma-ray burst (GRB), shown as a black circle (Greiner et al. 2015; Kann et al. 2016). Interaction-powered SNe, including SNe Ibn, can also show short rise times and high peak luminosities (e.g., Ofek et al. 2010; Hosseinzadeh et al. 2017).

Here we present an analysis of intermediate Palomar Transient Factory (iPTF) 16asu, a transient with a peak magnitude intermediate between SLSNe and ordinary $\mathrm{SNe}$ $\left(M_{\mathrm{g}}=-20.4 \mathrm{mag}\right)$ and an extremely rapid (4.0-day) rise to peak; shown as a red star in Figure 1. These characteristics place iPTF 16asu in a neighboring but unique part of transient phase space to the objects analyzed in Arcavi et al. (2016) and Drout et al. (2014). We present the photometric and spectroscopic observations of iPTF 16asu in Section 2, analyze the light curve and spectra in Section 3 and Section 4, and discuss the host galaxy in Section 5. We discuss the feasibility of several physical explosion mechanisms and energy sources for iPTF 16asu in Section 6 and summarize our findings in Section 7.

Throughout this paper, we assume a flat $\Lambda \mathrm{CDM}$ cosmology with $\Omega_{\mathrm{M}}=0.286$ and $H_{0}=69.6 \mathrm{~km} \mathrm{~s}^{-1} \mathrm{Mpc}^{-1}$.
Table 1

Log of iPTF 16asu Photometric Observations

\begin{tabular}{|c|c|c|c|c|}
\hline $\begin{array}{l}\text { Observation } \\
\text { Date } \\
\text { (MJD) }\end{array}$ & $\begin{array}{c}\text { Phase }^{\mathrm{a}} \\
\text { (rest- } \\
\text { frame days) }\end{array}$ & Filter & $\begin{array}{c}\text { Magnitude }^{\mathrm{b}} \\
\text { (AB) }\end{array}$ & Telescope \\
\hline 57508.32 & -12.50 & $g$ & $>20.61$ & $\mathrm{P} 48$ \\
\hline 57510.27 & -10.87 & $g$ & $>20.89$ & P48 \\
\hline 57510.30 & -10.84 & $g$ & $>20.80$ & P48 \\
\hline 57510.33 & -10.82 & $g$ & $>20.91$ & P48 \\
\hline 57511.26 & -10.03 & $g$ & $>20.78$ & P48 \\
\hline 57511.29 & -10.00 & $g$ & $>20.72$ & P48 \\
\hline 57511.32 & -9.98 & $g$ & $>20.53$ & P48 \\
\hline 57512.26 & -9.18 & $g$ & $>21.05$ & P48 \\
\hline 57512.29 & -9.16 & $g$ & $>20.82$ & P48 \\
\hline 57512.32 & -9.14 & $g$ & $>21.09$ & P48 \\
\hline 57513.25 & -8.35 & $g$ & $>20.96$ & P48 \\
\hline 57513.28 & -8.33 & $g$ & $>20.96$ & P48 \\
\hline 57513.31 & -8.30 & $g$ & $>20.72$ & P48 \\
\hline 57519.26 & -3.29 & $g$ & $20.43 \pm 0.13$ & P48 \\
\hline 57519.29 & -3.27 & $g$ & $>20.29$ & P48 \\
\hline 57519.32 & -3.24 & $g$ & $>20.01$ & P48 \\
\hline 57520.25 & -2.46 & $g$ & $19.80 \pm 0.12$ & P48 \\
\hline 57520.28 & -2.43 & $g$ & $19.69 \pm 0.08$ & P48 \\
\hline 57521.26 & -1.61 & $g$ & $19.34 \pm 0.09$ & P48 \\
\hline 57521.29 & -1.58 & $g$ & $19.25 \pm 0.09$ & P48 \\
\hline 57521.32 & -1.56 & $g$ & $19.28 \pm 0.09$ & P48 \\
\hline 57525.40 & 1.88 & $g$ & $19.38 \pm 0.09$ & P60 \\
\hline 57527.34 & 3.51 & $g$ & $19.51 \pm 0.07$ & P60 \\
\hline 57535.33 & 10.24 & $g$ & $20.43 \pm 0.09$ & P60 \\
\hline 57538.34 & 12.78 & $g$ & $20.87 \pm 0.05$ & P60 \\
\hline 57540.30 & 14.42 & $g$ & $21.01 \pm 0.07$ & P60 \\
\hline 57544.21 & 17.72 & $g$ & $21.49 \pm 0.08$ & P60 \\
\hline 57545.25 & 18.60 & $g$ & $21.48 \pm 0.11$ & P60 \\
\hline 57545.26 & 18.61 & $g$ & $21.14 \pm 0.09$ & P60 \\
\hline 57546.31 & 19.49 & $g$ & $21.69 \pm 0.13$ & P60 \\
\hline 57551.35 & 23.73 & $g$ & $>22.09$ & P60 \\
\hline 57560.26 & 31.24 & $g$ & $>20.29$ & P60 \\
\hline 57580.20 & 48.03 & $g$ & $>21.69$ & P60 \\
\hline 57581.23 & 48.90 & $g$ & $>21.19$ & P60 \\
\hline 57584.24 & 51.43 & $g$ & $>21.49$ & P60 \\
\hline 57587.18 & 53.91 & $g$ & $>21.69$ & P60 \\
\hline 57587.88 & 54.50 & $g$ & $>23.09$ & TNG \\
\hline 57527.33 & 3.51 & $r$ & $19.60 \pm 0.09$ & P60 \\
\hline 57535.32 & 10.23 & $r$ & $20.19 \pm 0.07$ & P60 \\
\hline 57540.27 & 14.40 & $r$ & $20.34 \pm 0.04$ & P60 \\
\hline 57541.18 & 15.17 & $r$ & $20.40 \pm 0.12$ & P60 \\
\hline 57541.18 & 15.17 & $r$ & $20.36 \pm 0.07$ & P60 \\
\hline 57544.19 & 17.70 & $r$ & $20.55 \pm 0.05$ & P60 \\
\hline 57544.25 & 17.75 & $r$ & $20.55 \pm 0.04$ & P60 \\
\hline 57544.26 & 17.76 & $r$ & $20.37 \pm 0.03$ & P60 \\
\hline 57545.23 & 18.58 & $r$ & $20.65 \pm 0.07$ & P60 \\
\hline 57545.23 & 18.58 & $r$ & $20.61 \pm 0.09$ & P60 \\
\hline 57546.29 & 19.47 & $r$ & $20.64 \pm 0.05$ & P60 \\
\hline 57551.32 & 23.71 & $r$ & $20.89 \pm 0.13$ & P60 \\
\hline 57554.24 & 26.17 & $r$ & $21.09 \pm 0.15$ & P60 \\
\hline 57554.25 & 26.17 & $r$ & $21.00 \pm 0.12$ & P60 \\
\hline 57560.24 & 31.22 & $r$ & $>20.83$ & P60 \\
\hline 57570.22 & 39.62 & $r$ & $21.73 \pm 0.20$ & P60 \\
\hline 57573.21 & 42.14 & $r$ & $22.06 \pm 0.14$ & P60 \\
\hline 57577.25 & 45.55 & $r$ & $>21.13$ & P60 \\
\hline 57580.19 & 48.02 & $r$ & $>21.53$ & P60 \\
\hline 57581.22 & 48.89 & $r$ & $>21.13$ & P60 \\
\hline 57584.23 & 51.42 & $r$ & $>20.03$ & P60 \\
\hline 57587.17 & 53.90 & $r$ & $>21.03$ & P60 \\
\hline 57587.90 & 54.52 & $r$ & $23.01 \pm 0.15$ & TNG \\
\hline 57593.21 & 58.99 & $r$ & $>21.73$ & P60 \\
\hline
\end{tabular}


Table 1

(Continued)

\begin{tabular}{|c|c|c|c|c|}
\hline $\begin{array}{l}\text { Observation } \\
\text { Date } \\
\text { (MJD) }\end{array}$ & $\begin{array}{c}\text { Phase }^{\mathrm{a}} \\
\text { (rest- } \\
\text { frame days) }\end{array}$ & Filter & $\begin{array}{c}\text { Magnitude }{ }^{b} \\
(A B)\end{array}$ & Telescope \\
\hline 57596.21 & 61.51 & $r$ & $>21.43$ & P60 \\
\hline 57525.40 & 1.88 & $i$ & $19.57 \pm 0.14$ & P60 \\
\hline 57527.33 & 3.51 & $i$ & $19.66 \pm 0.07$ & P60 \\
\hline 57535.33 & 10.24 & $i$ & $19.87 \pm 0.05$ & P60 \\
\hline 57538.33 & 12.77 & $i$ & $20.09 \pm 0.05$ & P60 \\
\hline 57540.28 & 14.41 & $i$ & $20.29 \pm 0.06$ & P60 \\
\hline 57544.20 & 17.71 & $i$ & $20.46 \pm 0.05$ & P60 \\
\hline 57544.21 & 17.72 & $i$ & $20.43 \pm 0.05$ & P60 \\
\hline 57544.26 & 17.77 & $i$ & $20.43 \pm 0.06$ & P60 \\
\hline 57545.24 & 18.59 & $i$ & $20.48 \pm 0.11$ & P60 \\
\hline 57545.25 & 18.59 & $i$ & $20.46 \pm 0.10$ & P60 \\
\hline 57546.29 & 19.48 & $i$ & $20.68 \pm 0.08$ & P60 \\
\hline 57551.33 & 23.72 & $i$ & $>20.84$ & P60 \\
\hline 57554.25 & 26.18 & $i$ & $20.82 \pm 0.16$ & P60 \\
\hline 57554.26 & 26.19 & $i$ & $20.73 \pm 0.12$ & P60 \\
\hline 57560.25 & 31.23 & $i$ & $>20.55$ & P60 \\
\hline 57570.23 & 39.63 & $i$ & $>21.25$ & P60 \\
\hline 57573.21 & 42.15 & $i$ & $>21.75$ & P60 \\
\hline 57580.20 & 48.03 & $i$ & $>20.64$ & P60 \\
\hline 57581.22 & 48.89 & $i$ & $>21.14$ & P60 \\
\hline 57584.23 & 51.43 & $i$ & $>20.05$ & P60 \\
\hline 57584.26 & 51.45 & $i$ & $>20.75$ & P60 \\
\hline 57587.17 & 53.90 & $i$ & $>21.25$ & P60 \\
\hline 57587.89 & 54.51 & $i$ & $23.07 \pm 0.16$ & TNG \\
\hline 57593.22 & 58.99 & $i$ & $>20.95$ & P60 \\
\hline 57596.21 & 61.51 & $i$ & $>21.14$ & P60 \\
\hline 57527.29 & 3.47 & $V$ & $>19.48$ & Swift \\
\hline 57527.29 & 3.47 & $B$ & $19.45 \pm 0.2$ & Swift \\
\hline 57527.29 & 3.47 & $u$ & $19.6 \pm 0.14$ & Swift \\
\hline 57527.29 & 3.47 & UVW1 & $20.52 \pm 0.14$ & Swift \\
\hline 57527.29 & 3.47 & UVW2 & $21.8 \pm 0.19$ & Swift \\
\hline 57527.29 & 3.47 & UVM2 & $21.27 \pm 0.14$ & Swift \\
\hline 57534.33 & 9.4 & $V$ & $>18.95$ & Swift \\
\hline 57534.33 & 9.4 & $B$ & $>19.61$ & Swift \\
\hline 57534.33 & 9.4 & $u$ & $>20.37$ & Swift \\
\hline 57534.33 & 9.4 & UVW1 & $>21.46$ & Swift \\
\hline 57534.33 & 9.4 & UVW2 & $>22.46$ & Swift \\
\hline 57534.33 & 9.4 & UVM2 & $>22.48$ & Swift \\
\hline 57541.19 & 9.4 & $V$ & $>18.96$ & Swift \\
\hline 57541.19 & 9.4 & $B$ & $>19.86$ & Swift \\
\hline 57541.19 & 9.4 & $u$ & $>20.68$ & Swift \\
\hline 57541.19 & 9.4 & UVW1 & $>21.78$ & Swift \\
\hline 57541.19 & 9.4 & UVW2 & $>22.60$ & Swift \\
\hline 57541.19 & 9.4 & UVM2 & $>22.56$ & Swift \\
\hline
\end{tabular}

Notes.

${ }^{\text {a }}$ Phase is in rest-frame days relative to bolometric maximum light.

${ }^{\mathrm{b}}$ Corrected for Galactic extinction.

(This table is available in its entirety in machine-readable form.)

\section{Observations}

\subsection{Intermediate Palomar Transient Factory Discovery}

iPTF 16asu was discovered by the intermediate Palomar Transient Factory (Law et al. 2009; Cao et al. 2016; Masci et al. 2017) and was first detected in data taken with the 48." Samuel Oschin Telescope at the Palomar Observatory (P48) on 2016 May 11.26 UT (UT dates are used throughout this paper) at coordinates R.A. $=12^{\mathrm{h}} 59^{\mathrm{m}} 09^{\mathrm{s}} \cdot 28$, decl. $=+13^{\circ} 48^{\prime} 09^{\prime \prime} \cdot 2$

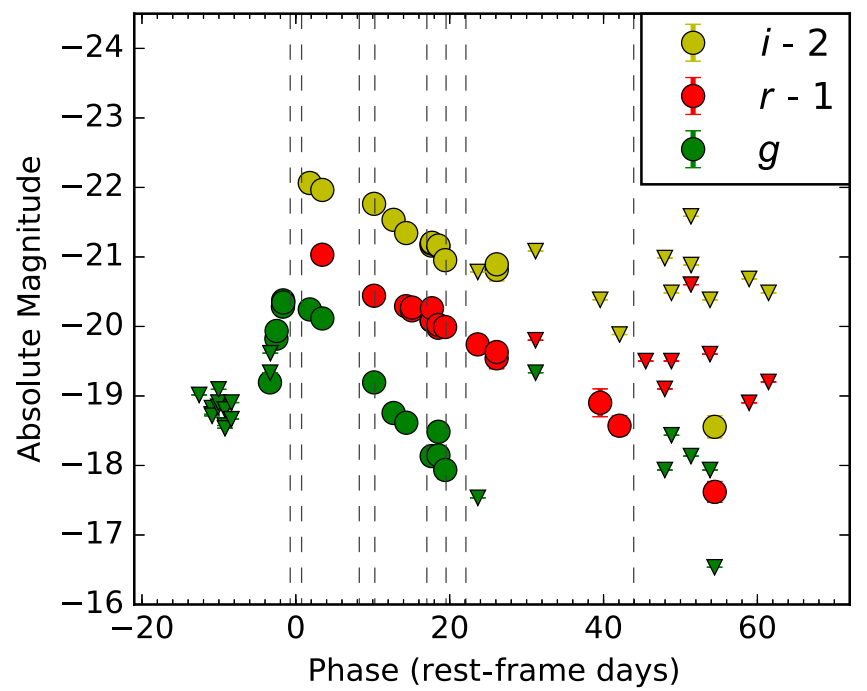

Figure 2. Light curves of iPTF 16asu in $g, r$, and $i$ filters. As indicated in the legend, the $r$ and $i$ band data have been offset for clarity. Triangles denote nondetections. Dashed lines indicate times of spectroscopic observations.

(J2000.0) and at a magnitude of $g=20.54$ mag. We obtained a spectrum with the Double Beam Spectrograph (DBSP; Oke \& Gunn 1982) on the 200" Hale Telescope at the Palomar Observatory (P200) on 2016 May 14.3 that shows a blue continuum and narrow $\mathrm{H} \alpha$ and [O III] lines from the host galaxy, setting the redshift to $z=0.187$. A later spectrum taken on 2016 June 04 by the Deep Imaging Multi-object Spectrograph (DEIMOS; Faber et al. 2003) on the $10 \mathrm{~m}$ Keck II Telescope on 2016 Jun 04 shows SN features consistent with an SN Ic-BL. The spectroscopic evolution is discussed in Section 4.

\subsection{Photometry}

iPTF 16asu was detected in a nightly cadence $g$ band experiment with iPTF, and we therefore have P48 data covering the time up to explosion as well as the early rise. Subsequent photometry was obtained with the automated 60". telescope at Palomar (P60; Cenko et al. 2006) in the gri bands. Host-subtracted point-spread function photometry was obtained using the Palomar Transient Factory Image Differencing and Extraction pipeline (Masci et al. 2017) on the P48 images and the FPipe SEDM presented in Fremling et al. (2016) on the P60 images using Sloan Digital Sky Survey (SDSS; SDSS Collaboration et al. 2016) images as templates and also calibrating to SDSS. Our last photometric observation came from the $3.58 \mathrm{~m}$ Telescopio Nazionale Galileo (TNG) and was processed through the FPipe. The photometry was corrected for Galactic extinction following Schlafly \& Finkbeiner (2011), with $E(B-V)=0.029 \mathrm{mag}$, and all magnitudes in this paper are reported in the AB system. Table 1 lists all photometric data, shown in Figure 2.

\subsection{Spectroscopy}

We obtained a sequence of eight low-resolution spectra for iPTF 16asu using the DBSP on P200, the Andalucia Faint Object Spectrograph and Camera (ALFOSC) on the $2.56 \mathrm{~m}$ Nordic Optical Telescope (NOT), the Device Optimized for the Low Resolution (DOLORES) on TNG, the Low-resolution Imaging Spectrometer (LRIS; Oke et al. 1995) on Keck I, and 
Table 2

Log of iPTF 16asu Spectroscopic Observations

\begin{tabular}{|c|c|c|c|c|c|c|c|c|}
\hline Observation Date & $\begin{array}{c}\text { Phase }^{\mathrm{a}} \\
\text { (rest-frame days) }\end{array}$ & Instrument & Grating & Filter & $\begin{array}{l}\text { Wavelength } \\
(\AA)\end{array}$ & $\begin{array}{c}\text { Resolution } \\
(\AA)\end{array}$ & $\begin{array}{c}\text { Exp. Time } \\
\text { (s) }\end{array}$ & Airmass \\
\hline 2016 May 14.30 & -0.73 & P200+DBSP & $600 / 4000$ & None & 3101-9199 & 1.30 & 600 & 1.21 \\
\hline 2016 May 16.06 & +0.75 & $\mathrm{NOT}+\mathrm{ALFOSC}$ & GRISM 4 & None & $3478-9662$ & 3.35 & 2400 & 1.35 \\
\hline 2016 May 24.97 & +8.25 & TNG+DOLORES & LR-B + LR-R & None & $3315-10330$ & 2.65 & 2100 & 1.09 \\
\hline 2016 May 27.36 & +10.27 & P200+DBSP & $600 / 4000$ & None & $3600-10237$ & 1.30 & 1800 & 1.69 \\
\hline 2016 Jun 04.39 & +17.03 & Keck 2+DEIMOS & 600ZD & GG455 & 4550-9649 & 0.65 & 1000 & 1.29 \\
\hline 2016 Jun 07.36 & +19.53 & Keck 1+LRIS & $400 / 3400,400 / 8500$ & None & $3072-10285$ & 1.55 & 950 & 1.17 \\
\hline 2016 Jun 10.42 & +22.11 & Keck 1+LRIS & $400 / 3400,400 / 8500$ & None & $3101-10290$ & 1.55 & 980 & 1.71 \\
\hline 2016 Jul 06.30 & +43.92 & Keck 1+LRIS & $400 / 3400,400 / 8500$ & None & $3067-10289$ & 1.55 & 2400 & 1.30 \\
\hline 2017 Apr 29.39 & +294.04 & Keck 1+LRIS & $400 / 3400,400 / 8500$ & None & $3063-10318$ & 1.55 & 2400 & 1.02 \\
\hline
\end{tabular}

Note.

${ }^{\text {a }}$ Phase is in rest-frame days relative to the bolometric maximum light (MJD 57523.25).

the DEIMOS on the $10 \mathrm{~m}$ Keck II Telescope. The times of the spectra are marked as dashed lines in Figure 2, and details of the spectroscopic observations are given in Table 2. Spectra were reduced using standard procedures using IRAF ${ }^{18}$ and IDL, including wavelength calibration using arc lamps and flux calibration using standard stars. The spectroscopic sequences for iPTF 16asu is shown in Figure 3, and the spectroscopic properties are analyzed and discussed in Section 4. All spectra will be made available in the Weizmann Interactive Supernova Data Repository (Yaron \& Gal-Yam 2012).

\subsection{Radio Observations}

We observed the field of iPTF 16asu with the Karl G. Jansky Very Large Array (VLA) on two epochs (Program VLA/16B043; PI: A. Corsi). The first observation was carried out starting on 2016 June 13, 01:18:22 UT (MJD 57552), with the VLA in its B configuration. The second observation was carried out with the VLA in its A configuration, starting on 2017 January 10, 09:43:06 UT (MJD 57763). Both these observations were carried out in the $C$ band (nominal central frequency of $\approx 5 \mathrm{GHz}$ ), using the 8 bit configuration and $2 \mathrm{GHz}$ nominal bandwidth. On both epochs we used 3C286 as a bandpass and flux density calibrator and $\mathrm{J} 1300+1417$ as a phase calibrator. The total observing time was about $1 \mathrm{hr}$ (including calibration and overhead) per epoch.

VLA data were calibrated using the automated VLA calibration pipeline in CASA (McMullin et al. 2007). After visual inspection, additional flags were applied when needed. Images of the fields were produced using the CLEAN task (Högbom 1974).

We searched for a radio counterpart to iPTF 16asu within a $2^{\prime \prime}$ radius circle centered on the iPTF position of iPTF 16asu. No radio source was detected within this region down to a $3 \sigma$ limit of $\approx 17 \mu \mathrm{Jy}$ at $6.2 \mathrm{GHz}$ for both epochs.

\subsection{UV and X-Ray Observations}

At the time of the first spectrum, iPTF 16asu resembled a very young SLSN, with its already high luminosity and blue spectrum indicating a high temperature. We therefore triggered our Swift program for SLSNe (GI-1215281, PI: R. Lunnan), and three epochs of Swift UVOT (Roming et al. 2005) and

\footnotetext{
${ }^{18}$ IRAF is distributed by the National Optical Astronomy Observatory, which is operated by the Association of Universities for Research in Astronomy under a cooperative agreement with the National Science Foundation.
}

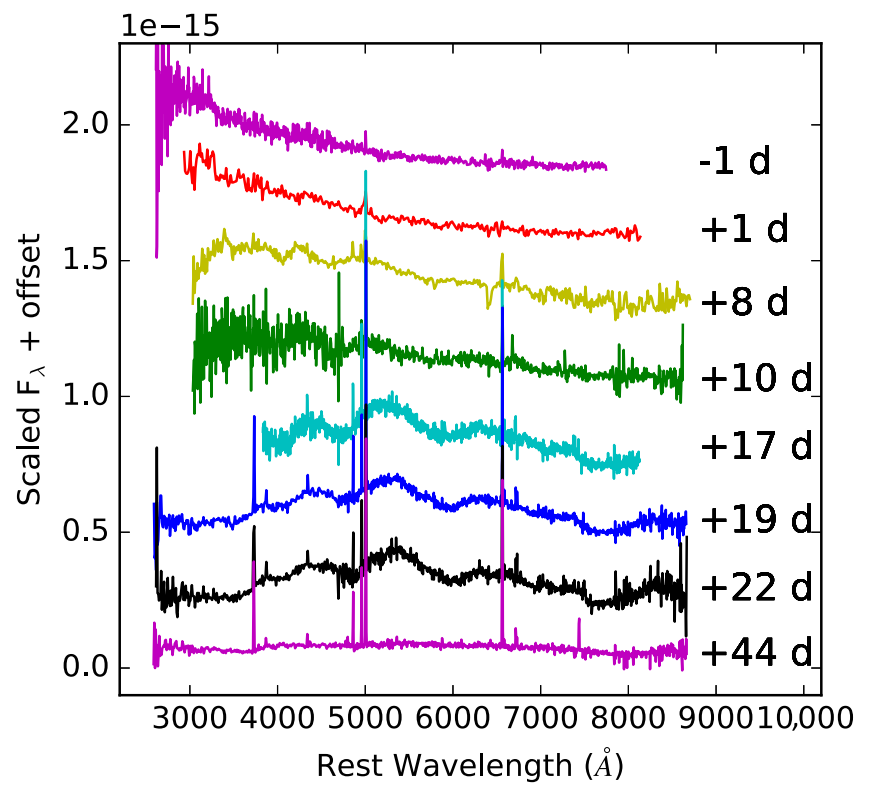

Figure 3. Sequence of observed spectra for iPTF 16asu. Phase in rest-frame days relative to $g$ band maximum is given to the right of each spectrum. The first two spectra show a featureless blue continuum, with broad SN features starting to be visible eight days after maximum. By day 17 , the spectrum has developed into that of an SN Ic-BL. Our last spectrum, taken 44 days after peak, is dominated by galaxy light. Galaxy narrow emission lines have not been removed. Spectra have been binned and arbitrarily scaled for display purposes. See Section 4 for details.

XRT (Burrows et al. 2005) data were obtained at phases corresponding to 7.4, 13.4, and 19.2 days after explosion (see Section 3.1 for the calculation of the explosion date).

We reduced the Swift data using the HEASoft package provided by NASA. ${ }^{19}$ UVOT photometry was performed using the task UVOTsource with an aperture of $5^{\prime \prime}$. iPTF 16asu was detected in all filters except the $V$ band in the first observation and undetected in all UVOT filters in the subsequent two epochs, due to the rapid fading of the SN. All UVOT photometry is listed in Table 1.

The XRT data were reduced with the Ximage software from the HEASoft package. No X-ray source was detected at the position of iPTF 16asu in either epoch. The $3 \sigma$ upper limits correspond to $5.6 \times 10^{-3}$ counts s$^{-1}, 2.9 \times 10^{-3}$ counts s$^{-1}$,

\footnotetext{
${ }^{19}$ http://heasarc.nasa.gov/lheasoft/
} 
and $3.9 \times 10^{-3}{\text { counts } \mathrm{s}^{-1} \text {, respectively. Using WebPIMMS }}^{20}$ and assuming a Galactic $\mathrm{nH}$ of $2.2 \times 10^{20} \mathrm{~cm}^{-2}$, we find that $1 \times 10^{-3}$ counts $\mathrm{s}^{-1}$ corresponds to $3.76 \times 10^{-14} \mathrm{erg} \mathrm{cm}^{-2} \mathrm{~s}^{-1}$ (unabsorbed; $0.3-10 \mathrm{keV}$ ), assuming a power-law model with an index of 2 . At a redshift of $z=0.1874$, our X-ray count limits translates to flux limits of $2.5 \times 10^{43} \mathrm{erg} \mathrm{s}^{-1}$, $1.1 \times 10^{43} \mathrm{erg} \mathrm{s}^{-1}$, and $1.5 \times 10^{43} \mathrm{erg} \mathrm{s}^{-1}$, respectively.

\subsection{Search for Associated GRBs}

We searched the Gamma-Ray Coordinates Network archives for any announced GRBs consistent with the location and bestfit explosion time of iPTF 16asu (Section 3.1). No announced GRB was consistent with the location and time of iPTF 16asu or when extending the search to include bursts detected between the last iPTF nondetection and the first detection of iPTF 16asu. However, our analysis of the Konus-Wind data (KW; Aptekar et al. 1995) reveals that a weak burst was detected by $\mathrm{KW}$ in the waiting mode (with a time resolution of 2.944 s) on 2016 May 10.41, which is consistent with our bestfit explosion time of 2016 May $10.53 \pm 0.17$ days (see Section 3.1). The burst was observed by the KW S2 detector pointing the northern ecliptic hemisphere (nothing was seen in the opposite S1 detector), which is also consistent with the position of iPTF 16asu, but the burst source position cannot be constrained more precisely from the KW data.

The burst emission was significant in the two softest KW

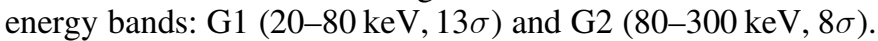
The burst light curve shows a single emission episode with a duration of $126 \mathrm{~s}\left(T_{50}=56 \pm 11 \mathrm{~s}\right.$ and $T_{90}=100 \pm 11 \mathrm{~s}$, both measured in the $20-300 \mathrm{keV}$ energy band). Fitting the KW tree-channel time-integrated spectrum (measured from T0 to T0 $+126.592 \mathrm{~s}$ ) by a simple power law yields the photon index of $2.35_{-0.14}^{+0.18}, \chi^{2} /$ dof $=2.7 / 1$. From this fit, the burst had an energy fluence of $8.25_{-0.86}^{+1.60} \times 10^{-6} \mathrm{erg} \mathrm{cm}^{-2}$ and a $2.944 \mathrm{~s}$ peak energy flux, measured from $\mathrm{T} 0+73.6 \mathrm{~s}$, of $2.41_{-0.94}^{+1.02} \times 10^{-7} \mathrm{erg} \mathrm{cm}^{-2} \mathrm{~s}^{-1}$ (both in the $20-1200 \mathrm{keV}$ energy range). At the distance of iPTF 16asu, this fluence would correspond to an equivalent isotropic energy $E_{\text {iso }}$ of $8.2 \times 10^{50} \mathrm{erg}$. The fit with a power law with an exponential cutoff model yields only an upper limit on spectrum peak energy: $E_{\mathrm{p}}<67 \mathrm{keV}$.

During the KW burst, Swift was in the South Atlantic Anomaly and the position of iPTF 16asu was Earth-occulted. However, the position of iPTF 16asu was not occulted for Fermi (and six GBM detectors had incident angles of less than $60^{\circ}$ ). We analyzed the Fermi-GBM continuous data and found no emission in the $30-300 \mathrm{keV}$ band coincident with the $\mathrm{KW}$ burst. Given that the background of Fermi-GBM is considerably lower than KW, this implies that the KW burst came from a source Earth-occulted to Fermi and therefore is not related to iPTF 16asu.

We also searched for a possible GRB in the INTEGRALSPI-ACS (SPI-ACS; von Kienlin et al. 2003) data covering the $75-8000 \mathrm{keV}$ range and found no candidate event down to the 3 sigma level. Since KW and SPI-ACS were observing the whole sky during the interval of interest, upper limits on gamma-ray flux can be obtained. For the whole interval (excluding the KW burst), assuming a typical long GRB spectrum (the band function with $\alpha=-1, \beta=-2.5$, and

\footnotetext{
${ }^{20}$ http://heasarc.gsfc.nasa.gov/cgi-bin/Tools/w3pimms/w3pimms.pl
}

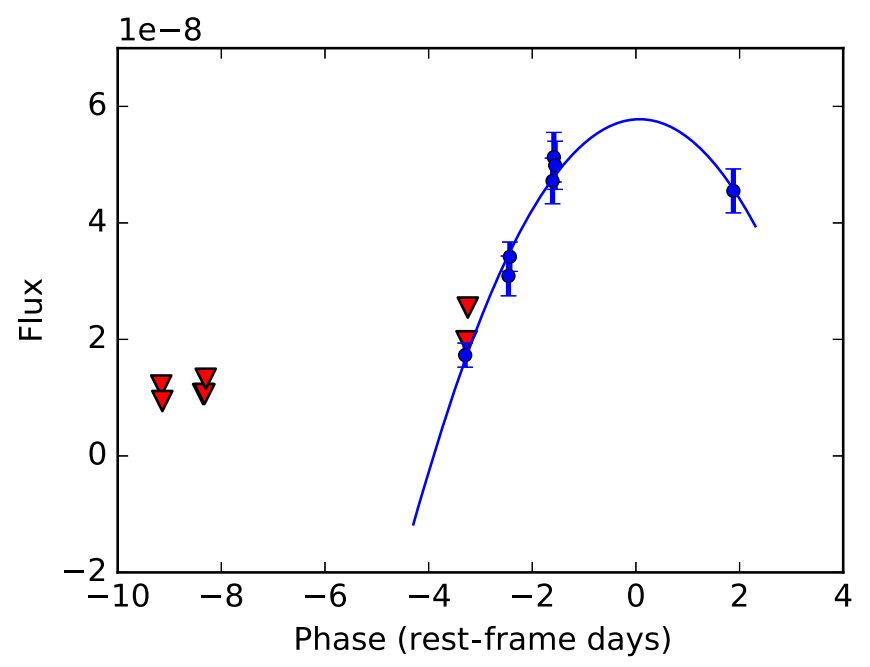

Figure 4. Early $g$ band light curve of iPTF 16asu, showing the rise of the light curve to peak. Red triangles denote $g$ band nondetections. The second-order polynomial best-fit line (blue) results in a calculated rise time of $3.97 \pm 0.19$ days. The equation of line of best fit is $y=-3.7 \times 10^{-9} x^{2}+4.8 \times 10^{-10} x+5.8 \times 10^{-8}$, where $x$ is the phase in days and $y$ is the flux $\left(F_{\lambda}\right)$ in arbitrary units.

$\left.E_{\mathrm{p}}=300 \mathrm{keV}\right)$, the corresponding $\mathrm{KW}$ and SPI-ACS limiting peak fluxes estimates are $\sim(1-4) \times 10^{-7} \mathrm{erg} \mathrm{cm}^{-2} \mathrm{~s}^{-1}$, both in the $10 \mathrm{keV}-10 \mathrm{MeV}$ band at 3-10 s time scales.

We conclude therefore that there is no statistically significant evidence for an $\mathrm{SN}$-associated GRB down to the threshold of $10^{-7} \mathrm{erg} \mathrm{cm}^{-2} \mathrm{~s}^{-1}$. The associated isotropic peak luminosity limit is $L_{\text {iso }} \lesssim 10^{49} \mathrm{erg} \mathrm{s}^{-1}$ and the total energy is $E_{\text {iso }} \lesssim 10^{50}$ erg (both calculated in the $10 \mathrm{keV}-10 \mathrm{MeV}$ energy range). Hence from these limits an accompanying lowluminosity GRB, like GRB $980425\left(L_{\text {iso }} \sim 5 \times 10^{46} \mathrm{erg} \mathrm{s}^{-1}\right.$, $E_{\text {iso }} \sim 10^{48} \mathrm{erg}$; Galama et al. 1998b), cannot be excluded. We return to discuss possible GRB models for iPTF 16asu in Section 6.3.

\section{Light Curve Analysis}

\subsection{Rise Time and Peak Luminosity}

The light curves of iPTF 16asu are shown in Figure 2. The rise and peak are only sampled in the $g$ band, so we fit a second-order polynomial to the $g$ band light curve near peak brightness to determine a best-fit explosion date, time of peak, and peak luminosity. The fit is shown in Figure 4, and the explosion and best-fit peak dates are MJD 57518.53 \pm 0.17 and MJD $57523.25 \pm 0.14$, respectively. Corresponding calendar dates are 2016 May 10.53 and 2016 May 15.25. Thus, the rise time (time of peak-time of explosion) is $3.97 \pm 0.19$ days in the rest frame. The last optical upper limit prior to the first detection was MJD 57513.31, setting an upper limit to the rise time of 9.94 days.

Using our series of spectra, we calculate $K$-corrections from the observed filters at $z=0.187$ to the rest-frame filters, listed in Table 3. At this redshift the observed gri corresponds most closely to the rest-frame $B V r$, and the wavelength coverage of our spectra allows us to also calculate $K$-corrections to the $u, g$, and $i$ filters. Applying this, we find that the time of peak corresponds to a peak absolute magnitude of $M_{\mathrm{B}}=-20.4$ mag (AB). 
Table 3

$K$-corrections Derived from Spectra

\begin{tabular}{|c|c|c|c|c|c|c|c|c|}
\hline $\begin{array}{l}\text { Phase } \\
\text { (rest-frame days) }\end{array}$ & $\underset{(\mathrm{mag})}{K_{\mathrm{rr}}}$ & $\begin{array}{c}K_{\mathrm{gr}} \\
(\mathrm{mag})\end{array}$ & $\begin{array}{c}K_{\mathrm{gg}} \\
(\mathrm{mag})\end{array}$ & $\begin{array}{c}K_{\mathrm{ug}} \\
(\mathrm{mag})\end{array}$ & $\begin{array}{c}K_{\mathrm{ii}} \\
(\mathrm{mag})\end{array}$ & $\underset{(\mathrm{mag})}{K_{\mathrm{ri}}}$ & $\begin{array}{c}K_{\mathrm{Bg}} \\
(\mathrm{mag})\end{array}$ & $\begin{array}{c}K_{\mathrm{Vr}} \\
(\mathrm{mag})\end{array}$ \\
\hline-0.73 & -0.368 & -0.041 & -0.270 & -0.194 & $\ldots$ & -0.173 & -0.192 & -0.269 \\
\hline+0.75 & -0.357 & -0.062 & -0.260 & -0.215 & $\cdots$ & -0.164 & -0.177 & -0.258 \\
\hline+8.25 & -0.136 & -0.286 & 0.056 & -0.372 & -0.285 & -0.206 & -0.078 & -0.175 \\
\hline+10.27 & -0.107 & -0.280 & 0.062 & -0.438 & -0.325 & -0.204 & -0.079 & -0.158 \\
\hline+17.03 & -0.109 & $\cdots$ & $\cdots$ & $\cdots$ & $\cdots$ & -0.199 & $\cdots$ & -0.121 \\
\hline+19.53 & -0.043 & -0.467 & 0.410 & -0.688 & -0.255 & -0.195 & 0.102 & -0.112 \\
\hline+22.11 & -0.050 & -0.487 & 0.422 & -0.768 & -0.214 & -0.187 & 0.127 & -0.110 \\
\hline
\end{tabular}

Notes.

${ }^{\mathrm{a}} K$-correction is defined as in Hogg et al. (2002), so that for filters $Q$ and $R, m_{\mathrm{R}}=M_{\mathrm{Q}}+D M+K_{\mathrm{QR}}$.

${ }^{\mathrm{b}}$ Phase is in rest-frame days relative to the bolometric maximum light (MJD 57523.25).
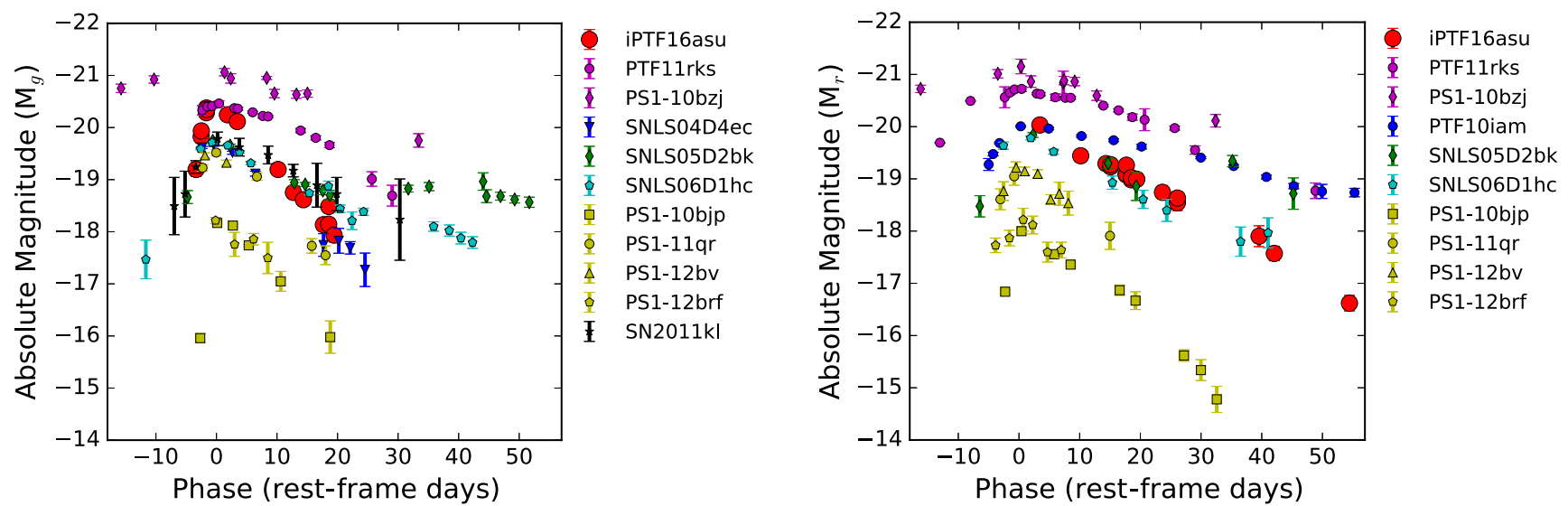

Figure 5. $g$-band (left) and $r$-band (right) light curves of iPTF 16asu (red) compared to other luminous and/or rapidly evolving transients from the literature. Filters have been chosen to correspond to approximately the same rest wavelengths. SLSNe from Inserra et al. (2013) and Lunnan et al. (2013) are shown in magenta; Arcavi et al. (2016) objects are shown in blue, cyan, and green; Drout et al. (2014) objects are shown in yellow; and SN 2011kl/GRB 111209A (Greiner et al. 2015) objects are shown in black.

\subsection{Light Curve Comparisons}

iPTF 16asu inhabits an unusual location in rise time versus luminosity parameter space (see Figure 1). In this section, we compare its light curve in more detail to objects in the literature that have been noted for their fast timescales and/or high luminosities. Unfortunately, $K$-corrections are not available for the majority of our comparison objects due to a lack of spectroscopic coverage. For the purposes of comparison, we corrected iPTF 16asu and all comparison objects for redshift using the following equations:

$$
\begin{gathered}
\lambda=\frac{\lambda_{\mathrm{obs}}}{(1+z)}, \\
M=m_{\mathrm{obs}}-5 \log _{10}\left(\frac{D_{L}}{10 \mathrm{pc}}\right)+2.5 \log _{10}(1+z) .
\end{gathered}
$$

We then chose filters with rest wavelengths as closely corresponding to those of iPTF16asu as possible in order to facilitate comparison. Comparing the approximation used in Equation (2) to the actual $K$-corrections calculated for iPTF 16asu (Table 3), we expect this approximation to introduce errors on the order of $0.1 \mathrm{mag}$. Figure 5 shows comparisons to the $g$ band (left) and $r$ band (right) light curves.

First we compare against the light curves of SNe noted for both their high luminosities and their rapid timescales. These include SN 2011kl (Greiner et al. 2015), an SN associated

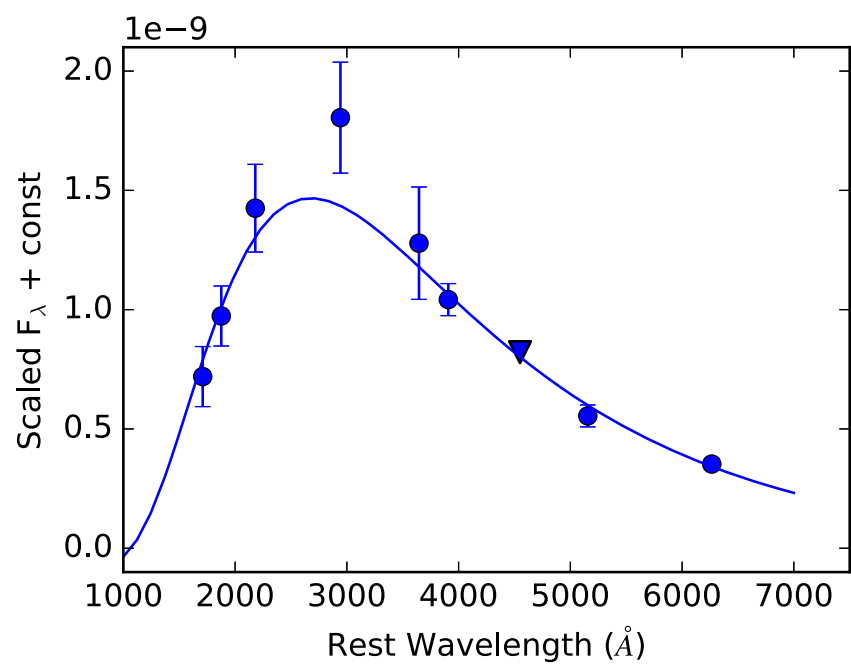

Figure 6. Blackbody fit of the Swift/UVOT and optical data, at phase +3 days. The triangle denotes a nondetection in the $V$ band. The best-fit estimates of the temperature and radius from the fit are $T=10,800 \pm 250 \mathrm{~K}$ and $R=(2.6 \pm 0.2) \times 10^{15} \mathrm{~cm}$.

with the ultralong gamma-ray burst GRB 111209A (plotted in black), and PTF 10iam (blue), SNLS04D4ec (blue), SNLS05D2bk (green), and SNLS06D1hc (cyan) from Arcavi et al. (2016). In the $g$ band as seen in Figure 5 (left), iPTF 16asu reaches a higher peak luminosity than these 

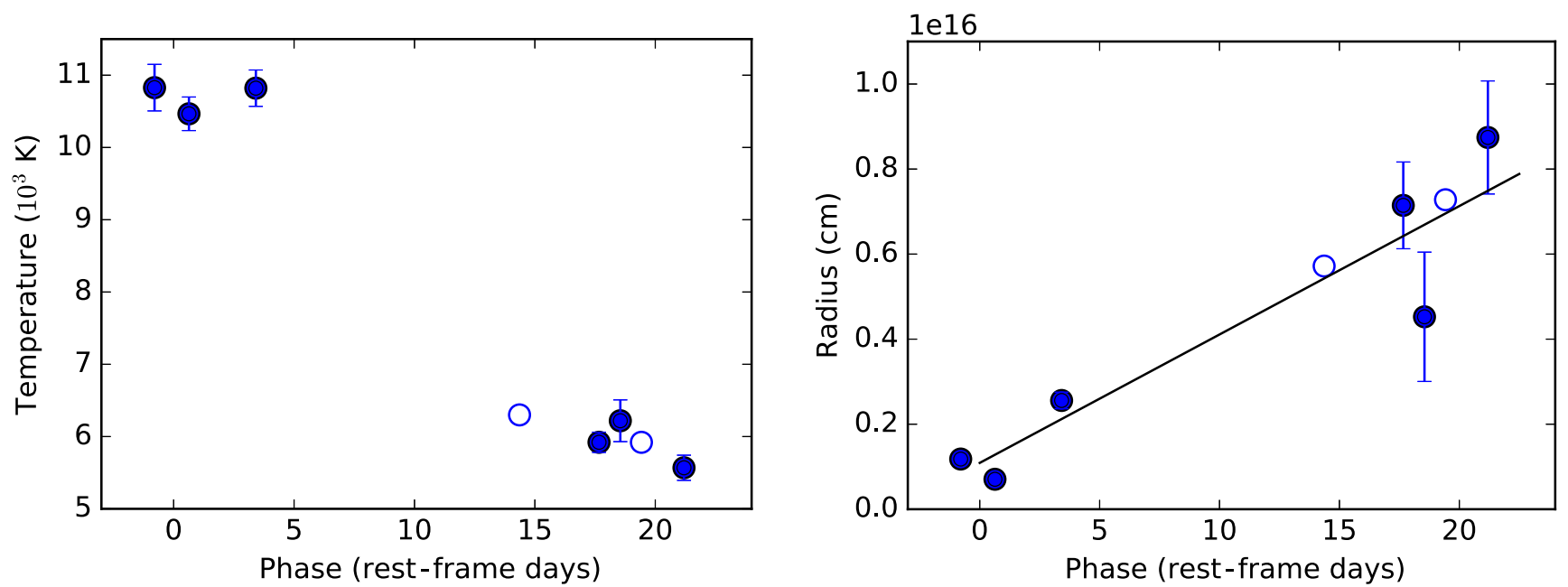

Figure 7. Blackbody temperature (left) and radius (right) as a function of time. We fit a blackbody to all epochs with photometry in at least three filters as well as to the earliest two spectra. The slope of the radius over time gives an estimated expansion velocity of $35,400 \pm 5350 \mathrm{~km} \mathrm{~s}^{-1}$. Open circles indicate points for which the covariance matrix would not converge to give error bars.

transients by over half a magnitude. Measuring from the restframe phase at $M_{\text {peak }-1 \mathrm{mag}}$ to $M_{\text {peak }+1 \mathrm{mag}}$, iPTF 16asu's timescale is about two times shorter with $\tau_{\text {peak }-1 \text { mag }}=10$ days. iPTF 16asu displays both a steeper rise and steeper decay than the Arcavi et al. (2016) objects and SN 2011kl in the $g$ band. However, iPTF 16asu resembles these objects more closely in the $r$ band, shown in Figure 5 (right). The peak $r$ band magnitude of iPTF 16asu is approximately the same as PTF 10iam, SNLS05D2bk, and SNLS06D1hc and the slope of decay runs nearly parallel to that of SNLS06D1hc. Although we have no data on the rise in the $r$ band, iPTF 16asu has a peak magnitude similar to that of to the Arcavi et al. (2016) objects and decays on the same timescale as SNLS06D1hc.

Next we compared the light curve to PS1-10bjp, PS1-11qr, PS1-12bv, and PS1-12brf, a sample of rapidly evolving transients from the Pan-STARRS1 Medium Deep Survey (Drout et al. 2014). The objects shown are the four most luminous objects from the "gold" sample and are plotted in yellow in Figure 5. They have rise times and decay slopes similar to those of iPTF 16asu but are much fainter. In the $g$ band, PS1-11qr and PS1-12bv are the brightest of the PanSTARRS1 objects, reaching a peak magnitude of about -19.5 mag; thus iPTF 16asu is a magnitude brighter at peak. As seen in Figure 5, the shape of iPTF 16asu's light curve is quite similar to that of PS1-10bjp and PS1-11qr. Early in the decay of iPTF 16asu, the slope is nearly parallel to that of PS110bjp; however, at late times PS1-10bjp decays more sharply than iPTF 16asu. Comparing these objects to the $r$ band data is less instructive because iPTF 16asu's rise was not captured in the $r$ band and most of the Pan-STARRS1 objects do not have late-time data.

Finally we compared iPTF 16asu to the SLSNe PTF11rks (Inserra et al. 2013) and PS1-10bzj (Lunnan et al. 2013), which are both on the lower-luminosity end of SLSNe. In the $g$ band, iPTF 16asu reaches about the same peak absolute magnitude as PTF11rks. In the $r$ band iPTF 16asu's peak luminosity is about 0.5 mag dimmer than that of PTF11rks. However, the SLSNe have timescales several times longer than that of iPTF 16asu, as seen by the much broader peaks. Thus, while iPTF 16asu reaches luminosities similar to those of some SLSNe, it evolves on a very different timescale. iPTF 16asu stands out as a unique and surprising event, even amongst similar transients from the literature.

\subsection{Blackbody Fits}

We fit a blackbody to all epochs where we have observations in at least three filters, using Scipy least-squares optimization routines (Jones et al. 2001) as well as to our two earliest spectra. Only the day with Swift/UVOT detections (+3 days past peak) has data in more than three filters. The fit to the Swift photometry is shown in Figure 6. From this fit we obtain $T=10,800 \pm 250 \mathrm{~K}$ and $R=(2.6 \pm 0.2) \times 10^{15} \mathrm{~cm}$. This corresponds to a total blackbody luminosity of $(6.4 \pm 1.6) \times 10^{43} \mathrm{erg} \mathrm{s}^{-1}$.

Figure 7 shows the resulting derived temperatures and radii at all epochs. The overall trends show a cooling blackbody temperature and an increasing radius. In fitting a straight line to the blackbody radii, we get a best-fit slope of $34,500 \pm 5400 \mathrm{~km} \mathrm{~s}^{-1}$, indicating high average velocities.

\subsection{Bolometric Light Curve}

We construct a pseudobolometric light curve for iPTF 16asu by summing the observed flux on days where we have observations in at least three filters. We integrate over the observed spectral energy distribution using trapezoidal integration, interpolating to the edges of the observed bands. Since this only accounts for the observed flux, it constitutes a strict lower limit on the true bolometric luminosity.

Pre-peak photometry is only available in the $g$ band so we approximate the rise of the pseudobolometric light curve by assuming a constant ratio of $g$ band flux to total flux, i.e., a constant bolometric correction. This assumption is equivalent to assuming that the temperature on the rise is constant and equal to the temperature measured from the earliest multiband data. Similarly, for the late-time observations with data only in the $r$ band we estimate the total flux by using the same bolometric correction as from the latest date with data in $\geqslant 3$ filters. 

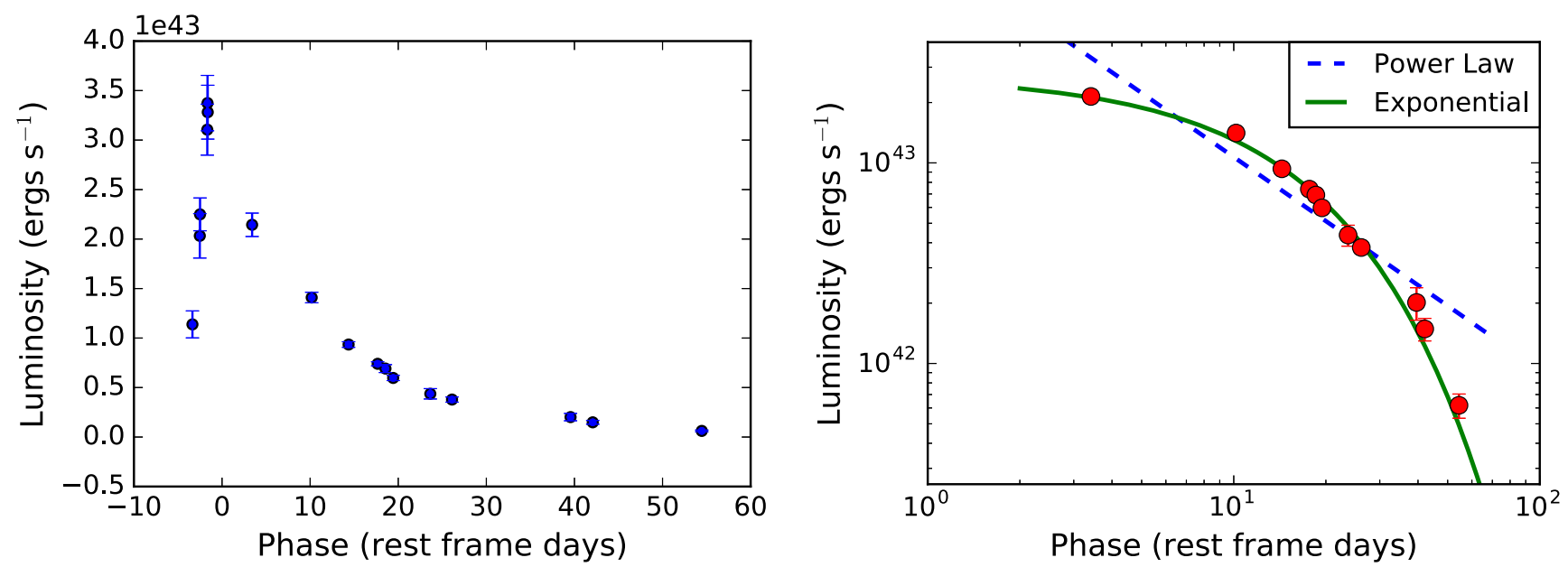

Figure 8. Left: Pseudobolometric light curve of iPTF 16asu. Luminosities obtained from data using the trapezoidal integration method. Right: Fit of the decline of iPTF 16asu's light curve to a power law and an exponential. The power law (dashed blue) has a best-fit of $L \propto t^{-1.06 \pm 0.14}$ and the exponential (solid green) decays are on a timescale of $\tau=13.56 \pm 0.56$ days. The light curve decline is well fit by an exponential.

We caution that in constructing a pseudobolometric light curve from optical data only, we are implicitly assuming that the ratio of optical to bolometric flux is approximately constant over the time period of interest. In particular, at late times as the effective temperature falls we would expect the near-IR (NIR) fraction of the bolometric luminosity to increase, which is unconstrained from observations, so assuming a constant bolometric correction is likely an underestimate. Unfortunately, NIR data are also not available for other fast-evolving SNe so we cannot use them as a basis for comparison. However, given that iPTF 16asu resembles a normal SN Ic-BL-like SN 1998bw from $\sim 20$ days past explosion onward (Sections 4 and 6.1), we expect its late-time evolution of the optical-to-bolometric flux ratio to resemble normal SNe Ic-BL. Comparing to the light curve samples analyzed in Lyman et al. (2014), we estimate that this adds an uncertainty on the level of $10 \%-20 \%$ at late times.

Figure 8 (left) shows the resulting pseudobolometric light curve. Using trapezoidal integration over time we calculate an estimated total radiated energy of $(4.0 \pm 0.6) \times 10^{49} \mathrm{erg}$ and a peak luminosity of $(3.4 \pm 0.3) \times 10^{43} \mathrm{erg} \mathrm{s}^{-1}$.

The shape of the decline of the bolometric light curve sheds light on what physical processes may be powering this event. Figure 8 (right) shows the best-fit power law and exponential fits to the post-peak light curve. Clearly the decline of the light curve does not follow a power law; however, it fits an exponential well. The power law has a best-fit decay of $L \propto t^{-1.06 \pm 0.14}$ and the exponential decays are on a timescale of $\tau=13.56 \pm 0.56$ days. The power-law decay parameter is similar to those found for the objects in Arcavi et al. (2016). Also similar, two of the four Arcavi et al. (2016) objects are better fit by an exponential. The implications of these results are discussed in Section 6.

\section{Spectroscopic Properties}

\subsection{Spectroscopic Evolution and Comparisons}

We obtained eight spectra of iPTF 16asu between 2016 May 14 and 2016 Jul 6. The spectra are shown in Figure 3. In this section, we look at the spectroscopic evolution in more detail and compare the spectroscopic properties of iPTF 16asu to similar objects from the literature.

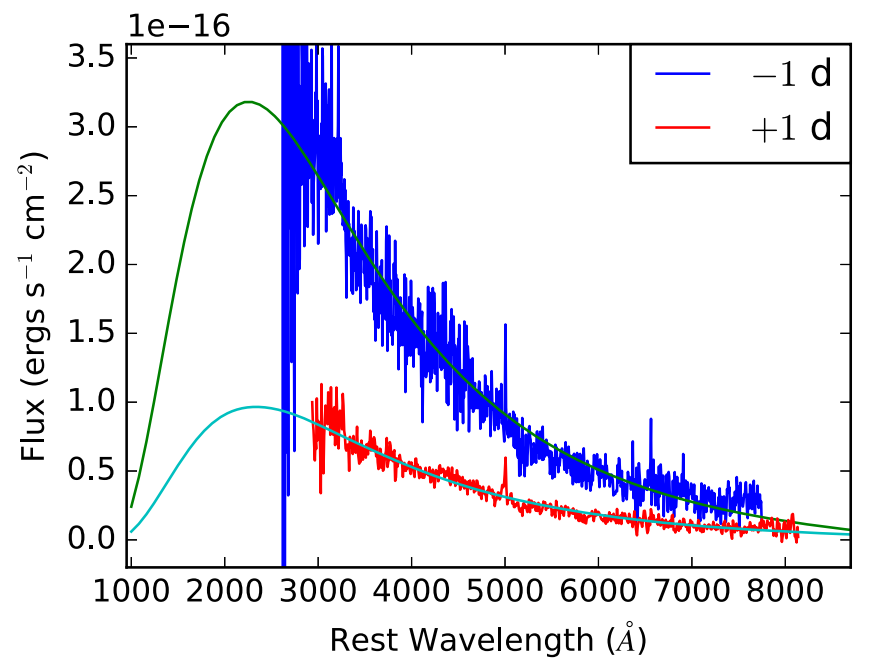

Figure 9. Blackbody fit of the two earliest spectra. The corresponding temperature and radius at phase -1 day are $T=10828 \pm 322 \mathrm{~K}$ and $R=(1.18 \pm 0.07) \times 10^{15} \mathrm{~cm}$. The corresponding temperature and radius at phase +1 day are $T=10,466 \pm 232 \mathrm{~K}$ and $R=(7.05 \pm 0.03) \times 10^{14} \mathrm{~cm}$.

The first two spectra, taken within a day before and after peak, show a featureless blue continuum with no discernible broad features. The spectrum is well fit by a blackbody, as shown in Figure 9. Such spectra dominated by blue continua have also been observed at early phases in other $\mathrm{SNe}$, typically while the luminosity is powered by the cooling of the stellar envelope following shock breakout (see, e.g., SN 1993J; Woosley et al. 1994; Matheson et al. 2000). Interestingly, the rapidly evolving SNe from Pan-STARRS1 (Drout et al. 2014) also showed featureless blue continua. Figure 10 shows a comparison of PS1-12bv at peak compared to iPTF 16asu at peak. Unfortunately, comparison at late times is not possible as there is no further follow-up spectroscopy on the PanSTARRS1 events. Based on the limited spectroscopic data available we cannot rule out that they were caused by the same phenomenon as iPTF 16asu.

The next two spectra, taken at phases 8 and 10 days past maximum, still show an underlying blue continuum but with broad features emerging. Such an evolution is reminiscent of 


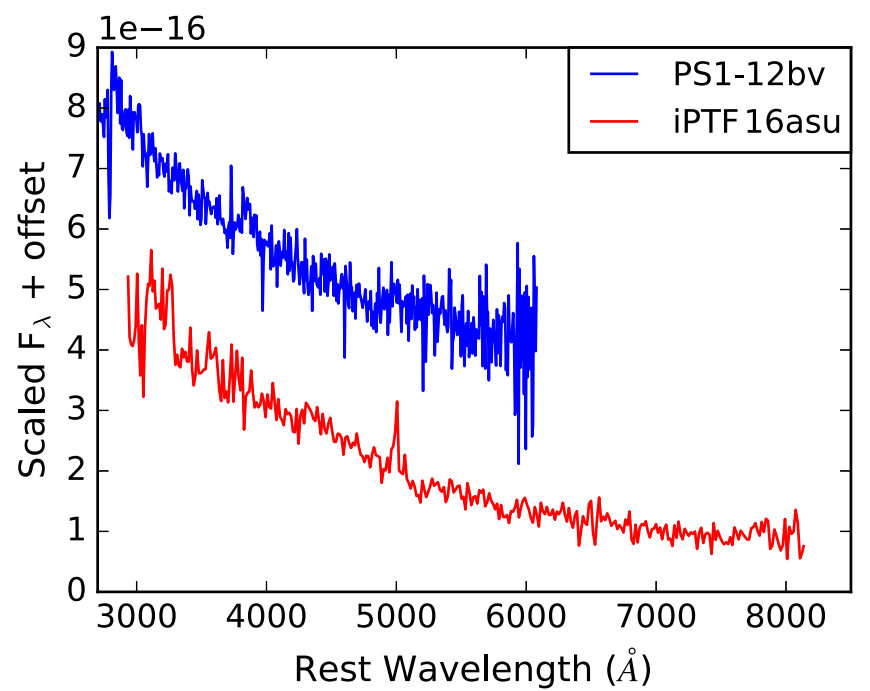

Figure 10. Spectrum of PS1-12bv (Drout et al. 2014) at +7 days after explosion compared to iPTF 16asu at +5 days after explosion. iPTF 16asu spectrum from NOT. Host galaxy narrow emission lines have not been removed - note that the feature at $\sim 5000 \AA$ in the iPTF 16 asu spectrum is a narrow [O III] $\lambda \lambda 4959,5007$ emission from the host galaxy that appears broadened here due to binning.

GRB SNe. To illustrate this we show a comparison to SN 2006aj/GRB 060218 (Modjaz et al. 2006) in Figure 11. SN 2006aj is of particular interest here because it is one of the few GRB SNe that would not be ruled out by our radio and X-ray limits. We discuss GRB models for iPTF 16asu in detail in Section 6.3.

The three spectra taken at phases $+17,+19$, and +22 days post maximum are dominated by distinct broad-line features, leading us to classify iPTF 16asu as an SN Ic-BL. Figure 12 shows a comparison of iPTF 16asu at +23 days after explosion (+19 days past peak) to SN 1998bw at +18 days after explosion, and features commonly identified in SNe Ic-BL are marked. Interestingly, the spectra of these events look very similar at roughly the same time after explosion, suggesting that iPTF 16asu may have a normal-timescale SN component hidden underneath the luminous and rapidly evolving peak.

It is also worth noting that the spectroscopic evolution of iPTF 16asu is different from the few objects in Drout et al. (2014) and Arcavi et al. (2016) with spectra at later phases: PS1-12bb showed a featureless continuum at phase +33 days, while PTF 10iam showed broad $\mathrm{H} \alpha$ emission at phase +28 days. This spectroscopic diversity suggests that there are likely multiple physical mechanisms giving rise to light curves in this part of the transient phase space.

Our final spectrum, taken at phase +44 days past peak, is dominated by host galaxy light. We discuss the host galaxy properties in Section 5.

\subsection{Velocities}

Measuring velocities from SNe Ic-BL spectra is challenging since the lines are often blended due to the high velocities. In addition, different lines can give different velocities because these elements are formed and found at different radii in the expanding ejected material. For iPTF 16asu we chose the strongest lines: the $\mathrm{Si}$ II $\lambda 6355 \AA$ line and the Fe II $\lambda 5169$ $\AA$ line.

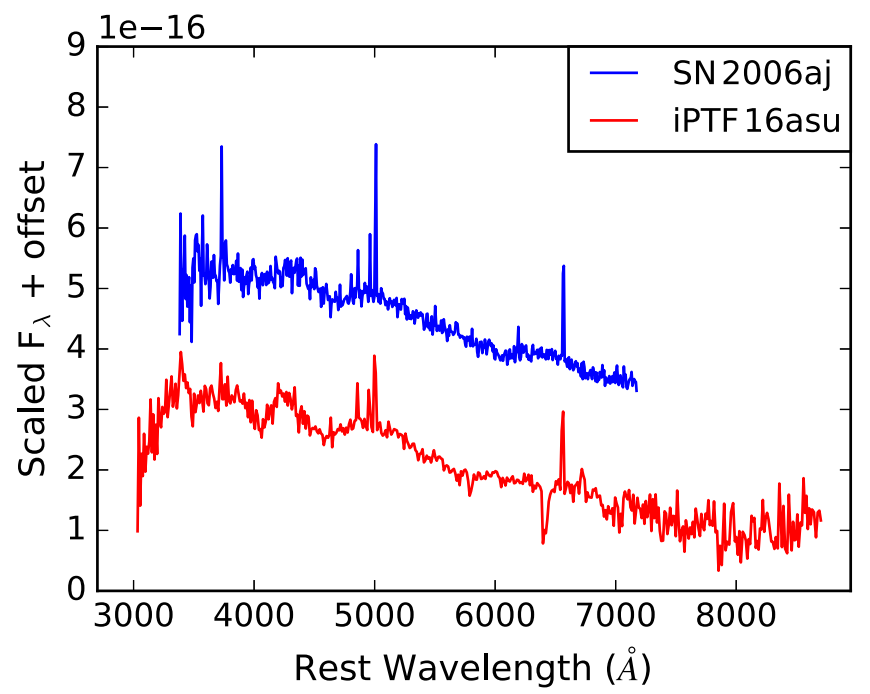

Figure 11. Spectrum of SN 2006aj (Modjaz et al. 2006) at +6 days after explosion compared to iPTF 16asu at +12 days after explosion. iPTF 16asu spectrum from TNG. Host galaxy narrow emission lines have not been removed.

In the case of the $\mathrm{Si}$ II $\lambda 6355 \AA$ line we fit a parabola to find the minimum of the broad absorption feature. The corresponding wavelength is then used to determine velocities using the relativistic Doppler shift. The measured velocities are listed in Table 4.

In the case of the Fe II $\lambda 5169 \AA$ line, similar to other $\mathrm{SNe}$ Ic-BL, this line is blended with the neighboring Fe II $\lambda 4924$ and Fe II $\lambda 5018$ lines. Thus we cannot simply fit the minimum of this feature to derive velocities. Instead we use the convolution method developed by Modjaz et al. (2016) and Liu et al. (2016) to extract velocities from the Fe II $\lambda 5169$ $\AA$ line. The measured velocities are listed in Table 4. Figure 13 shows the Fe II velocities from iPTF 16asu compared to the sample of SNe Ic and Ic-BL from Modjaz et al. (2016), with velocities derived using the same method (and code). The Fe II velocities we measure for iPTF 16asu are high compared to the objects in this sample, closest to the Fe II velocities of SNe Ic$\mathrm{BL}$ associated with GRBs. We note that phase in this figure is measured with respect to maximum light-if iPTF 16asu has a normal SN component hidden underneath the blue luminous peak, then the SN maximum will be later and iPTF 16asu will move left in this plot, but the basic conclusion that the velocities are comparable to SNe Ic-BL with associated GRBs will be unchanged.

\section{Host Galaxy}

The host galaxy of iPTF 16asu is detected in both the PTF templates and the SDSS images. The observed SDSS model magnitudes are $u^{\prime}=22.90 \pm 0.37 \mathrm{mag}, g^{\prime}=22.10 \pm$ $0.09 \mathrm{mag}, \quad r^{\prime}=21.82 \pm 0.11 \mathrm{mag}, \quad i^{\prime}=21.43 \pm 0.11 \mathrm{mag}$, and $z^{\prime}=21.25 \pm 0.28 \mathrm{mag}$. At a redshift of $z=0.1874$ this makes the host a dwarf galaxy with an absolute magnitude of $M_{g} \simeq-17.5$ mag. We use the FAST code (Kriek et al. 2009) to fit a galaxy model to the observed photometry using a Maraston (2005) stellar population synthesis model, assuming a Salpeter initial mass function and an exponential star formation history. The metallicity and extinction are constrained by our spectroscopic data, so we use the extinction derived from the Balmer decrement and a metallicity of 


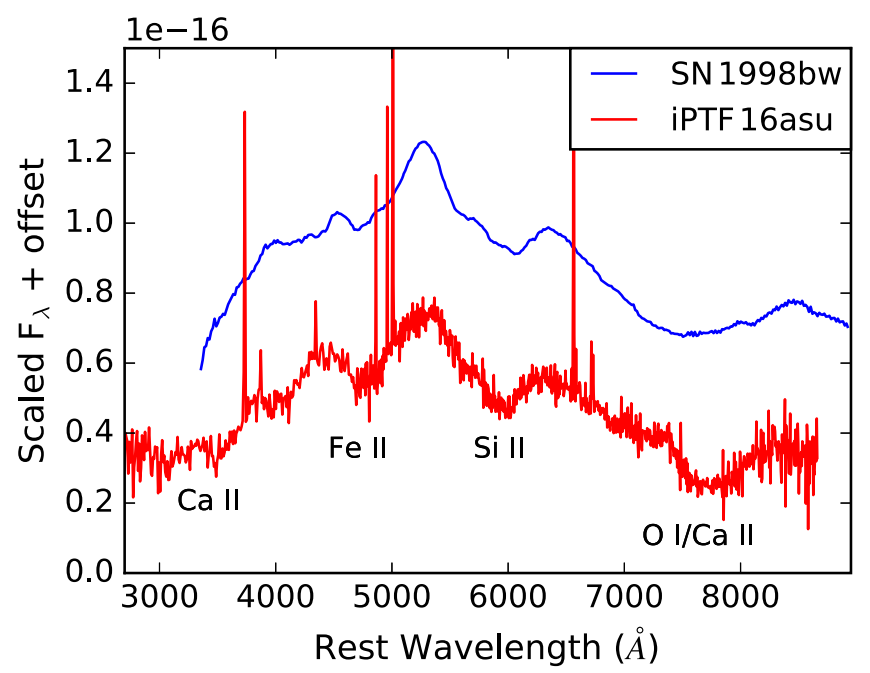

Figure 12. Spectrum of SN 1998bw (Patat et al. 2001) at +18 days after explosion compared with iPTF 16asu at +23 days after explosion. Features commonly identified in SNe Ic-BL are marked. iPTF 16asu spectrum from Keck 1+LRIS. Host galaxy narrow emission lines have not been removed.

$Z=0.5 Z_{\odot}$, which is the closest model grid value to our derived metallicity. With these assumptions, we find a best-fit stellar mass of $M_{*}=4.6_{-2.3}^{+2.0} \times 10^{8} M_{\odot}$ and a best-fit stellar population age of $5.0_{-4.6}^{+6.5} \times 10^{8} \mathrm{yr}$.

We obtained a host galaxy spectrum nearly a year after explosion, shown in Figure 14. We scale this galaxy spectrum to the SDSS photometry to account for slit losses, and measure the fluxes of the (unresolved) lines by fitting Gaussian profiles. The measured emission line fluxes are listed in Table 5.

We use the Balmer decrement to calculate the host galaxy extinction, assuming Case B recombination (Osterbrock 1989). We measure a $\mathrm{H} \alpha / \mathrm{H} \beta$ ratio of $3.5 \pm 0.2$, translating to a host extinction of $E(B-V)=0.22 \pm 0.06$, assuming a standard Milky Way extinction curve with $R_{V}=3.1 \quad$ (Cardelli et al. 1989). Using the extinction-corrected $H \alpha$ flux, we measure a star formation rate of $0.7 M_{\odot} \mathrm{yr}^{-1}$ (Kennicutt 1998). Given the stellar mass derived from the photometry, this corresponds to a specific star formation rate of $1.4 \mathrm{Gyr}^{-1}$.

We use pyMCZ (Bianco et al. 2016) to calculate the galaxy oxygen metallicity from the [O III], [O II], [N II], $\mathrm{H} \alpha$, and $\mathrm{H} \beta$ lines. $\mathrm{pyMCZ}$ is a Python-based implementation of up to 15 metallicity calibrators, updating the code given in Kewley \& Dopita (2002) and Kewley \& Ellison (2008) and with better treatment of statistical uncertainty from Monte Carlo sampling. While there is some scatter between the different strong-line metallicity estimators, they generally agree that the host galaxy of iPTF 16asu is low metallicity. For example, we find values of $12+\log (\mathrm{O} / \mathrm{H})$ to be $8.12_{-0.07}^{+0.04}$ on the Pettini \& Pagel (2004) O3N2 scale, $8.22_{-0.07}^{+0.18}$ on the McGaugh (1991) scale, and $8.39_{-0.05}^{+0.11}$ on the Kobulnicky \& Kewley (2004) $R_{23}$ scale, to name three commonly used indicators. Using a solar oxygen abundance of $12+\log (\mathrm{O} / \mathrm{H})=8.69 \pm 0.05 \quad$ (Asplund et al. 2009), this translates to a metallicity of $Z \simeq Z_{\odot} / 3$.

Taken together, the host galaxy of iPTF 16asu was a lowmass, low-metallicity, star-forming dwarf galaxy. Such an environment is not unusual for SNe Ic-BL, which in general are found in lower-metallicity galaxies than other strippedenvelope $\mathrm{SNe}$; for example, the median metallicity of $\mathrm{SN}$ Ic-BL hosts in the compilation of Sanders et al. (2012) was $12+\log (\mathrm{O} / \mathrm{H})=8.20$ on the Pettini \& Pagel (2004) O3N2 scale. Other rare transients, such as long GRBs and SLSNe, also show a preference for low-metallicity galaxies (e.g., Levesque et al. 2010; Lunnan et al. 2014; Perley et al. 2016). The high specific star formation rate and the strong [O III] $\lambda 5007 \AA$ line $\left(\mathrm{EW}_{5007} \simeq 87 \AA\right.$, rest-frame $)$ in particular is reminiscent of SLSN host galaxies (Leloudas et al. 2015). Interestingly, the same is not true for the rapidly evolving transients studied in Drout et al. (2014) and Arcavi et al. (2016): for both samples, the host galaxies were generally more massive galaxies near solar metallicity.

\section{Model Comparisons}

\subsection{Nickel Decay}

Most SNe Ic/Ic-BL are powered by the release of energetic photons from the radioactive decay of ${ }^{56} \mathrm{Ni}$ into ${ }^{56} \mathrm{Co}$ and finally ${ }^{56} \mathrm{Fe}$. Since the late-time spectra of iPTF 16asu look very similar to the spectra of other SNe Ic-BL (Section 4), we first consider whether the light curve of iPTF 16asu can be explained purely by the decay of ${ }^{56} \mathrm{Ni}$.

Using the equations from Section 2 of Lyman et al. (2016), we compare our pseudobolometric light curve from Section 3.4 to the theoretical model for a ${ }^{56} \mathrm{Ni}$ decay-powered light curve in Arnett (1982). The model takes two input parameters: diffusion time and ${ }^{56} \mathrm{Ni}$ mass. The ${ }^{56} \mathrm{Ni}$ mass predominantly affects the luminosity of the light curve, as a larger ${ }^{56} \mathrm{Ni}$ mass would indicate a larger total energy input and the diffusion time controls the timescale over which the energy diffuses out (i.e. width of the peak). Figure 15 shows the bolometric light curve of iPTF 16asu plotted against an Arnett (1982) model with the parameters $M_{\mathrm{Ni}}=0.55 M_{\odot}$ and $\tau_{\text {diff }}=1.5$ days, assuming an opacity of $\kappa=0.1 \mathrm{~cm}^{2} \mathrm{~g}^{-1}$. As seen in Figure 15 , the ${ }^{56} \mathrm{Ni}$ decay model does not fit both the sharp peak and the steep decay well, though we caution that the lack of NIR data could mean that our late-time bolometric light curve is systematically underestimated (Section 3.4).

An ejecta mass of $M_{\mathrm{ej}}=0.06 M_{\odot}$ was calculated using this diffusion time along with an estimate of the kinetic energy. Since our spectra near peak are featureless, and thus we cannot measure a velocity, we used the average velocity $\left(35,000 \mathrm{~km} \mathrm{~s}^{-1}\right)$ derived from the evolution of the blackbody radii to calculate this kinetic energy. The ejecta mass is notably about 10 times smaller than the amount of ${ }^{56} \mathrm{Ni}$ required to power this light curve, which is unphysical: the ${ }^{56} \mathrm{Ni}$ mass cannot be larger than the total ejecta mass, since it is necessarily part of the ejecta. Thus we rule out spherically symmetric radioactive ${ }^{56} \mathrm{Ni}$ decay as the dominant energy source for iPTF 16asu.

The Arnett model considered above assumes spherical symmetry and a central energy source, i.e., that all the nickel is in the center. Therefore we cannot rule out the possibility of ${ }^{56} \mathrm{Ni}$-powered models for iPTF 16asu in a highly mixed or strongly asymmetric scenario (e.g., a jet), though we note that assymmetry is not expected to have a large effect on the observed luminosity (Barnes et al. 2017). More sophisticated modeling is outside of the scope of this paper.

Although ${ }^{56} \mathrm{Ni}$ decay alone cannot explain the light curve of iPTF 16asu, it may still contribute. Figure 16 shows iPTF 16asu's light curve compared to other SNe Ic-BL from the literature. The light curve of SN 1998bw in the $g$ and $r$ bands is a good match to that of iPTF 16asu from $\sim 15$ to 
Table 4

Spectral Line Velocities of iPTF 16asu

\begin{tabular}{|c|c|c|c|c|}
\hline Observation Date & $\begin{array}{c}\text { Phase }^{\mathrm{a}} \\
\text { (rest-frame days) }\end{array}$ & $\begin{array}{l}\text { Fe II Velocity } \\
\left(1000 \mathrm{~km} \mathrm{~s}^{-1}\right)\end{array}$ & $\begin{array}{r}\text { Fe II Broadening } \\
\left(1000 \mathrm{~km} \mathrm{~s}^{-1}\right)\end{array}$ & $\begin{array}{c}\text { Si II Velocity } \\
\left(1000 \mathrm{~km} \mathrm{~s}^{-1}\right)\end{array}$ \\
\hline 2016 May 24.97 & +8.25 & $28.3_{-1.3}^{+1.1}$ & $5.5_{-1.2}^{+1.0}$ & \\
\hline 2016 May 27.36 & +10.27 & $29.5_{-1.4}^{+1.0}$ & $5.9_{-1.3}^{+1.0}$ & 23.3 \\
\hline 2016 Jun 07.36 & +19.53 & $21.6_{-0.4}^{+0.3}$ & $4.4_{-0.5}^{+0.4}$ & 19.2 \\
\hline 2016 Jun 10.42 & +22.11 & $22.0_{-1.3}^{+1.0}$ & $4.3_{-1.3}^{+1.3}$ & 16.8 \\
\hline
\end{tabular}

Note.

${ }^{\mathrm{a}}$ Phase is in rest-frame days relative to the bolometric maximum light (MJD 57523.25).

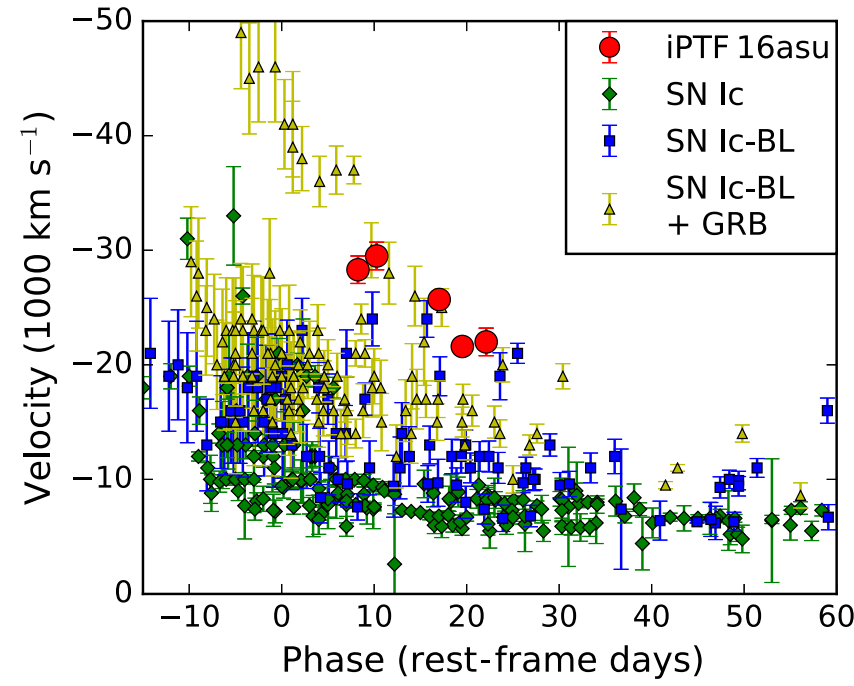

Figure 13. Velocity evolution of iPTF 16asu, measured from the Fe II $\lambda 5169$ $\AA$ line (red points), compared against literature data of $\mathrm{SNe}$ Ic (green diamonds), SNe Ic-BL (blue squares), and SNe Ic-BL (yellow triangles) associated with GRBs. Data from Modjaz et al. (2016).

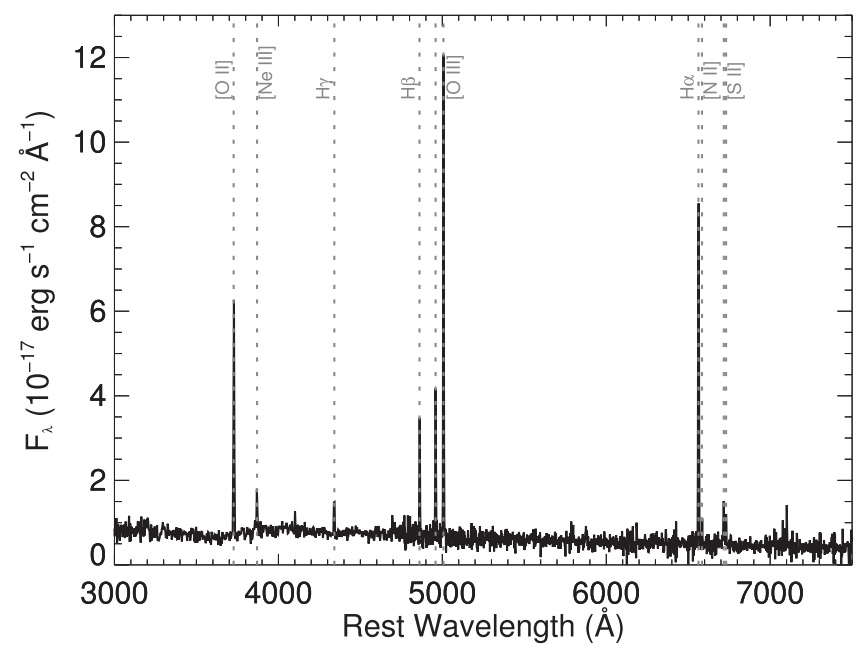

Figure 14. Spectrum of the host galaxy of iPTF 16asu, taken with Keck 1 + LRIS at $\sim 300$ days after the SN explosion. Strong galaxy emission lines are marked.

40 days, which interestingly also corresponds to the time when their spectra are very similar (Figure 12), suggesting that the light curve of iPTF 16asu could plausibly be dominated by a normal SN component at these times. Their late-time slopes deviate, mainly constrained by our last $r$ band point at 60 days
Table 5

Host Galaxy Emission Line Fluxes

\begin{tabular}{lc}
\hline \hline Line & $\begin{array}{c}\text { Flux } \\
\left(10^{-16} \mathrm{erg} \mathrm{s}^{-1} \mathrm{~cm}^{-2}\right)\end{array}$ \\
\hline$[\mathrm{O}$ II] 3727 & $3.50 \pm 0.10$ \\
{$[\mathrm{Ne}$ III] 3869 } & $0.62 \pm 0.08$ \\
$\mathrm{H} \gamma$ 4341 & $0.56 \pm 0.07$ \\
$\mathrm{H} \beta$ 4861 & $1.41 \pm 0.08$ \\
{$[\mathrm{O}$ III] 4959 } & $1.91 \pm 0.12$ \\
{$[\mathrm{O}$ III] 5007 } & $5.72 \pm 0.09$ \\
$\mathrm{H} \alpha$ 6563 & $5.01 \pm 0.09$ \\
[N II] 6583 & $0.24 \pm 0.10$ \\
[S II] 6717 & $0.66 \pm 0.13$ \\
[S II] 6731 & $0.41 \pm 0.14$ \\
\hline
\end{tabular}

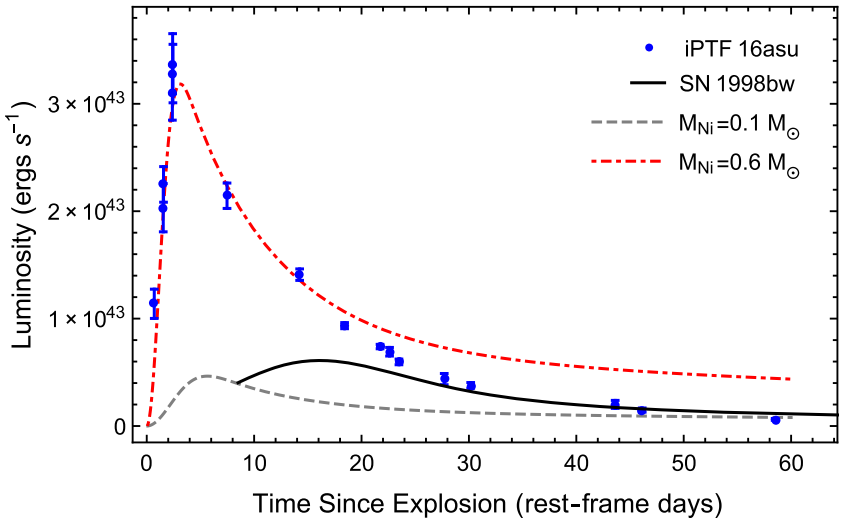

Figure 15. Nickel-powered model fit to the light curve of iPTF 16asu, following Arnett (1982) and Lyman et al. (2016). The red dot-dashed curve shows the model with the parameters $M_{\mathrm{Ni}}=0.55 M_{\odot}$ and $\tau_{\text {diff }}=1.5$ days. The dotted gray line shows the model constrained by the last point with the parameters $M_{\mathrm{Ni}}=0.1 M_{\odot}$ and $\tau_{\text {diff }}=3.7$ days. For comparison, the bolometric light curve using $B V(R I)_{\mathrm{c}}$ bands of SN 1998bw (Clocchiatti et al. 2011) is plotted in black. Attempting to fit the sharp luminous light curve with a ${ }^{56} \mathrm{Ni}$ model leads to an unphysical solution in which the derived ejecta mass is lower than the required nickel mass.

(Figure 16)-however, the decay rates of stripped-envelope $\mathrm{SNe}$ are heterogeneous and could be explained by differences in opacity and/or asymmetry, affecting the degree of gammaray trapping (Wheeler et al. 2015; Dessart et al. 2017). Fainter SNe Ic-BL such as SN 2006aj and SN 2002ap are below the light curve of iPTF 16asu at all times (Figure 16). Since the light curve shows only a single, smooth peak, any ${ }^{56} \mathrm{Ni}$ decay contribution to the total luminosity must be subdominant to whatever is powering the main peak.

Finally we note that other radioactive species, such as ${ }^{48} \mathrm{Cr}$ and ${ }^{52} \mathrm{Fe}$, have been proposed to power a class of fast-and-faint 

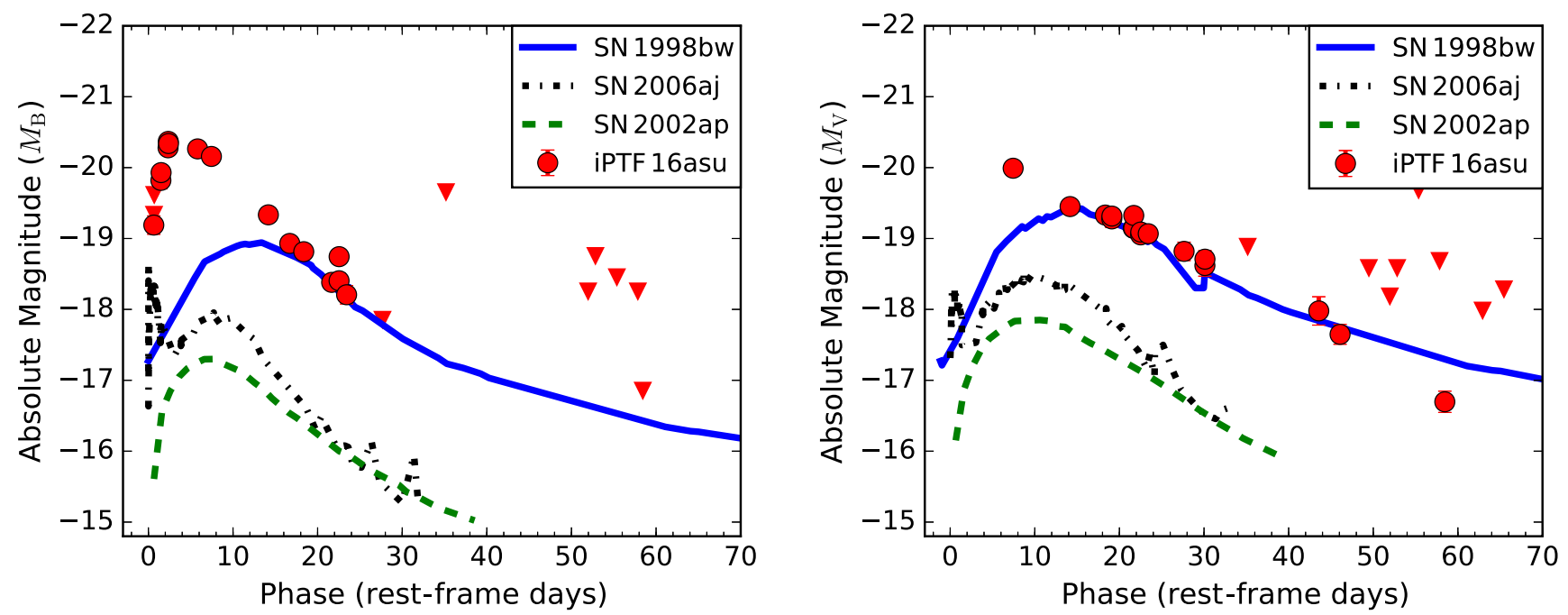

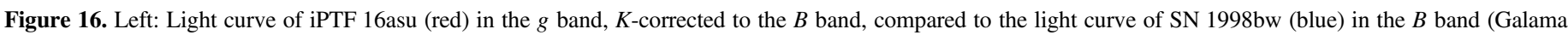

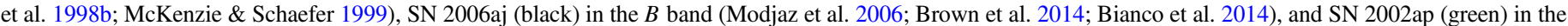
$B$ band (Foley et al. 2003). Right: Light curve of iPTF 16asu in the $r$ band, $K$-corrected to the $V$ band, compared to the same SNe, all in the $V$ band.

thermonuclear transients from He-shell detonations, so-called. Ia SNe (Bildsten et al. 2007; Shen et al. 2010). However, given that iPTF 16asu is 3-5 magnitudes brighter than these models predict, the spectrum at peak is blue and featureless without the expected strong Ti II features, and the late-time spectrum is an excellent match to $\mathrm{SNe}$ Ic-BL, suggesting a core-collapse explosion, we do not consider these models relevant for iPTF 16asu.

\subsection{Magnetar}

During the core collapse of a massive star, a highly magnetized $\left(B \approx 10^{14}-10^{15} \mathrm{G}\right)$ rapidly spinning neutron star called a magnetar can be formed. As the newborn magnetar spins down, rotational energy is released and can significantly boost the luminosity of the SN if the spin-down time of the magnetar is comparable to the diffusion time through the ejecta (e.g., Kasen \& Bildsten 2010; Woosley 2010; Metzger et al. 2015). Magnetar models have been suggested to explain highly luminous transients, including many SLSNe as well as SN 2011kl (Greiner et al. 2015; Bersten et al. 2016). iPTF 16asu has a luminosity similar to that of to SN 2011kl and some relatively low-luminosity SLSNe (Figures 1 and 5), so we examine whether a magnetar model can explain the peculiar light curve of iPTF 16asu.

As described in Kasen \& Bildsten (2010), the hydrodynamic simulations for their magnetar model makes the simplifying assumption that all of the injected energy is thermalized spherically at the base of the ejecta (ignoring the possibility of anisotropic jet-like injection). They further assume homologous expansion, a shallow power-law structure for interior density, and the dominance of radiation pressure. An expanding bubble with a thin shell of swept-up ejecta and a low-density interior are formed due to central overpressure in the SN remnant but rarely affect the outer layers of the $\mathrm{SN}$ ejecta. At late times the energy injected by the magnetar continues to heat the ejecta, as in ${ }^{56} \mathrm{Ni}$ decay, but it is no longer dynamically important. This process significantly affects the SN light curve.

The shape of the light curve in magnetar models depends on three parameters: $P$, the initial spin period; $B$, the strength of the magnetic field; and $\tau_{\text {diff }}$, the diffusion timescale that is

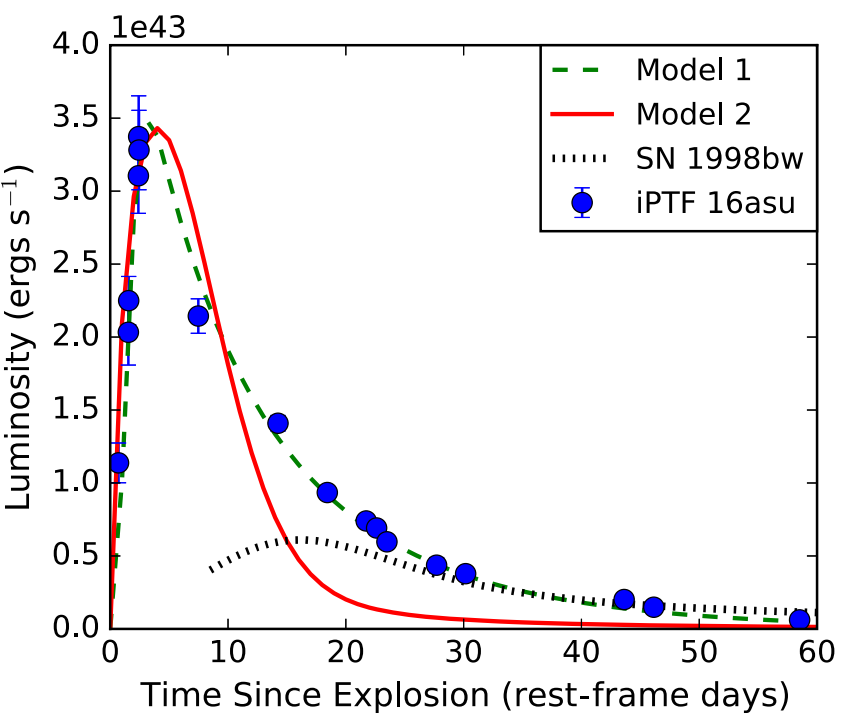

Figure 17. Model 1, the magnetar model best fit to the full light curve, shown in dashed green. Parameters are $P=9.95 \mathrm{~ms}, B=3.15 \times 10^{14}$ Gauss, and $\tau_{\text {diff }}=1.8$ days. Model 2, the magnetar model best fit with constrained $M_{\mathrm{ej}}=$ $1 M_{\odot}$, shown as a red line. Parameters are $P=6.0 \mathrm{~ms}, B=4.4 \times 10^{15}$ Gauss, and $\tau_{\text {diff }}=8.7$ days. The pseudobolometric light curve using $B V(R I)_{\mathrm{c}}$ bands of SN 1998bw (Clocchiatti et al. 2011) is plotted in dotted black to demonstrate how ${ }^{56} \mathrm{Ni}$ decay may power the late-time light curve.

proportional to $M_{\mathrm{ej}}^{1 / 2}$. Using the magnetar model fitting code from Kangas et al. (2017) we recover the parameters $B=(3.25 \pm 0.44) \times 10^{14}$ Gauss, $P=10.40 \pm 0.62 \mathrm{~ms}$, and $\tau_{\text {diff }}=1.59 \pm 0.06$ days. Manually tweaking the parameters slightly to obtain a better visual fit, we show the resulting fit to the bolometric light curve in Figure 17 with the parameters $P=9.95 \mathrm{~ms}, B=3.15 \times 10^{14} \mathrm{G}$, and $\tau_{\text {diff }}=1.8$ days, and assuming an opacity of $\kappa=0.1 \mathrm{~cm}^{2} \mathrm{~g}^{-1}$. As done in the ${ }^{56} \mathrm{Ni}$ model, the diffusion time and average velocity from the blackbody fits are used to calculate an ejecta mass of $M_{\mathrm{ej}}=0.09 M_{\odot}$. The parameters allow for the energy and the timescale to essentially be tuned separately, making the magnetar model quite flexible and generating a tight fit to both the peak and the decay of the bolometric light curve. 

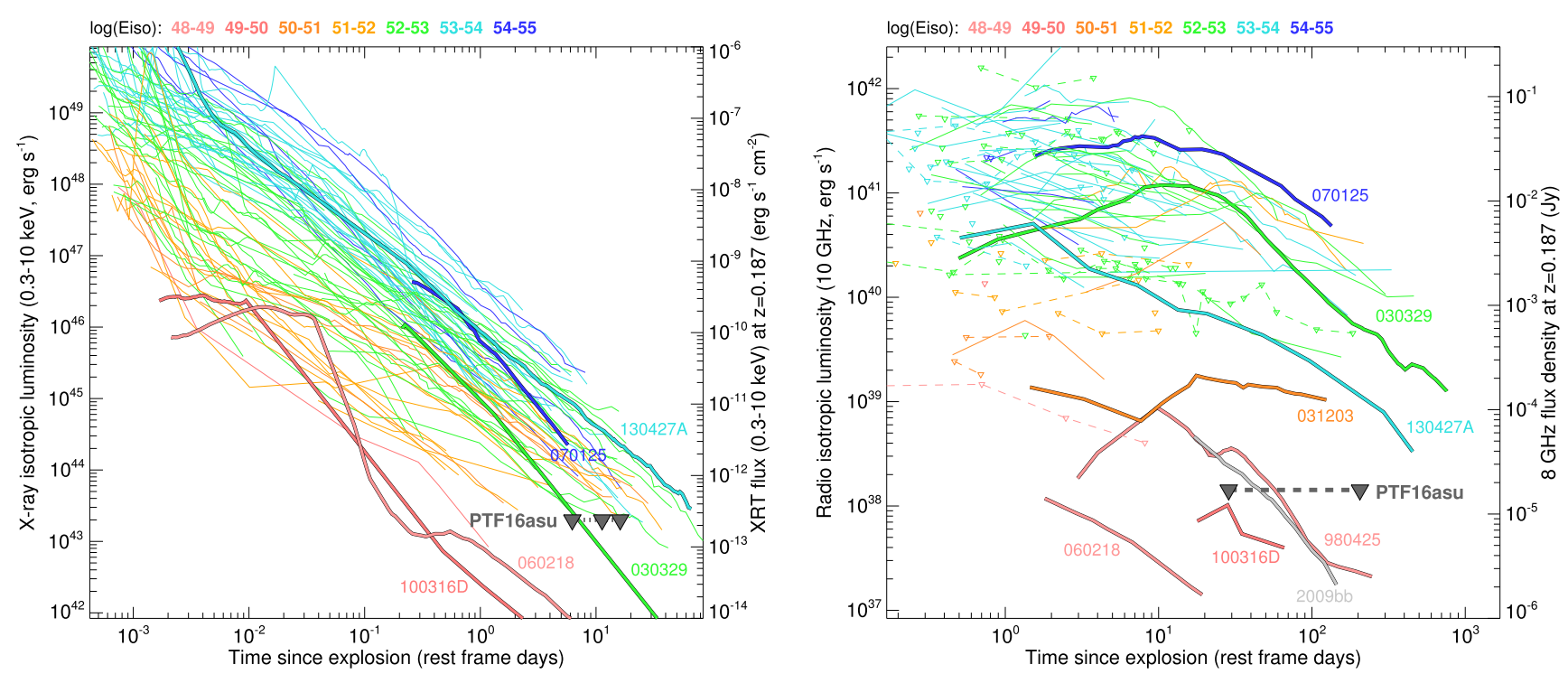

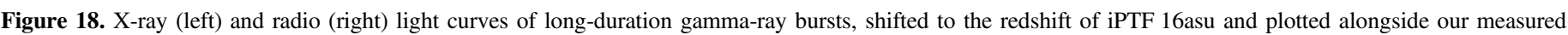

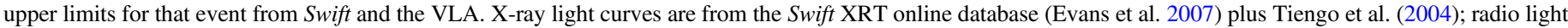

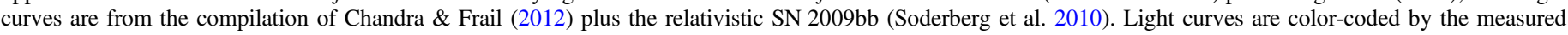

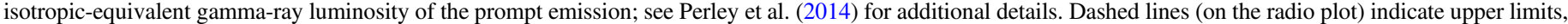

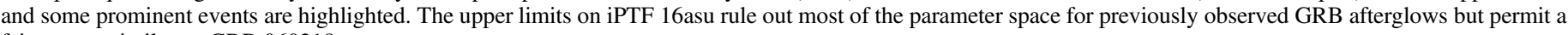
faint event similar to GRB 060218 .

Although the magnetar model produces a light curve that fits iPTF 16asu, the derived ejecta mass of our best fit is very low. Arcavi et al. (2016) derived similarly small ejecta masses for their rapidly rising $\mathrm{SNe}$ events, which caused them to conclude that the magnetar model was unlikely, while Greiner et al. (2015) concluded that a magnetar was a likely explanation for SN $2011 \mathrm{kl}$ despite their low derived ejecta mass. For an SN Ic-BL caused by the core collapse of a massive star, a magnetar model with such a low ejecta mass would require an extreme stripping scenario to reduce the core mass. Furthermore, the Kasen \& Bildsten (2010) magnetar model was tuned to an ejecta mass of $M_{\mathrm{ej}}=5 M_{\odot}$ and it is not clear that the assumptions of this model would remain valid in this low-mass regime.

Another way for a magnetar model to produce a fast timescale peak, similar to that of iPTF 16asu, is to use a small period and a high magnetic field, thereby decreasing the spin-down time. When constraining the ejecta mass to be $M_{\mathrm{ej}}=1.0 M_{\odot}$, we find the best-fit parameters $P=6.0 \mathrm{~ms}$, $B=4.4 \times 10^{15}$ Gauss, and $\tau_{\text {diff }}=8.7$ days. This fit is shown as a red line in Figure 17 and can also reproduce the fast rise and luminous peak of iPTF 16asu. However, this model declines too quickly to explain the entire light curve, so one would need a two-component model (e.g., with the late-time powered by ${ }^{56} \mathrm{Ni}$, as was considered by Bersten et al. 2016 for SN 2011kl). Thus despite the compelling light curve fit, we conclude that a magnetar model is unlikely to be the sole power source of iPTF 16asu but remains a candidate for powering the peak emission if the late time light curve is powered by ${ }^{56} \mathrm{Ni}$.

\subsection{Off-axis $G R B$}

Long GRBs are often associated with SNe Ic-BL, though not every SN Ic-BL has an accompanying GRB (see, e.g., Woosley \& Bloom 2006 for a review of the GRB-SN connection). GRBs are extremely energetic, relativistic, and highly beamed explosions characterized by an initial flash of gamma-rays followed by an "afterglow" of radiation typically seen at wavelengths ranging from the X-ray to the radio. iPTF 16asu's spectra and velocities are similar to those of SNe Ic-BL associated with GRBs (Section 4, Figures 11 and 12), so we examine whether the excess blue emission at peak could be explained as a GRB afterglow.

Nondetections of iPTF 16asu in the X-ray and radio strongly constrain the allowable GRB parameter space. The upper limits from three epochs of Swift data are shown in the left panel of Figure 18. While data at earlier times would have been more constraining, the upper limits rule out the bulk of observed X-ray afterglows with $E_{\text {iso }}>10^{52} \mathrm{erg}$; however, weak or offaxis GRBs are not excluded by the X-ray data alone. Similarly, the right panel shows the upper limits from our two epochs of VLA data. As evident from this figure, we can exclude a radio counterpart to iPTF 16asu as luminous as SN 1998bw or SN 2009bb, but we cannot exclude a lower-luminosity and/or faster-evolving radio counterpart such as SN 2006aj and SN 2010bh. If iPTF 16asu is associated with a GRB, then these limits suggest that it must be a faint $\left(E_{\mathrm{iso}}<10^{50} \mathrm{erg}\right)$ event. These constraints are consistent with the analysis from all-sky gamma-ray monitors (Section 2.6), as an on-axis burst at the distance of iPTF 16asu with $\left(E_{\text {iso }}>10^{50} \mathrm{erg}\right)$ would have been seen by KW or SPI-ACS.

The most unusual characteristic of iPTF 16asu is its abrupt four-day rise time in the optical. Such a rise time is extremely short in an SN context but would be unprecedentedly long in an SN-GRB context, even though optical afterglow light curves do sometimes show a rise (e.g., GRB 970508; Galama et al. 1998a). To explain the shape of the optical light curve as a GRB afterglow, we therefore consider off-axis GRB models.

From the New York University Afterglow Library data set of off-axis long GRBs at an observed wavelength of $3000 \AA$ $\left(10^{15} \mathrm{~Hz}\right)$, the models can reproduce a three- to six-day rise for an observer angle of between $23^{\circ}$ and $17^{\circ}$, respectively (van Eerten et al. 2010). The data set assumes a jet energy of $2 \times 10^{51} \mathrm{erg}$, a jet half opening angle of $11^{\circ} .5$, and a homogeneous circumburst number density of $1 \mathrm{~cm}^{-3}$. The 
parameters for an observer angle of $17^{\circ}$ produce a light curve with roughly the same peak magnitude as iPTF 16asu; however, in changing to an observed wavelength of $30 \mathrm{~mm}$ $(10 \mathrm{GHz})$, these parameters produce a radio light curve that is orders of magnitude brighter than our radio limits. Similarly, considering the low-energy models from van Eerten \& MacFadyen (2011), we find that parameters that satisfy the radio limits are inconsistently faint in the optical. The coarse grid of parameters used in van Eerten et al. (2010) does not allow us to make precise comparisons to their model but indicates that while a four-day optical rise could be constructed, our optical light curve and radio upper limits cannot be simultaneously satisfied by current models. A more thorough exploration of energy and density parameter space than is available in these model grids is necessary to determine whether GRB models can account for both the bright optical emission and the lack of X-ray and radio emission.

A similar conclusion can be reached by comparing the observed spectral properties of iPTF 16asu to typical GRB afterglows, which are well described by synchrotron radiation resulting in both a light curve and a spectrum consisting of several power-law segments with associated indices (e.g., Sari et al. 1998). If the featureless blue spectra of iPTF 16asu are due to a GRB afterglow then we expect the spectrum to follow a power law $\left(F_{\nu} \propto \nu^{-\beta}\right)$, with typical values of the power-law index $\beta$ around $0.5-0.6$ (e.g., Kann et al. 2010). Fitting our first spectrum (at +3 days after explosion) with a power law, we find a best-fit index of $\beta=-0.5$, i.e., $F_{\nu} \propto \nu^{+0.5}$, which is inconsistent with a GRB-like spectrum. In contrast, the spectrum is well fit by a blackbody (Figure 6). Similarly, if we compare our earliest X-ray upper limit to the corresponding point on the $r$ band light curve, then we derive a limit on the optical- to-X-ray spectral index $\beta_{\mathrm{OX}}>1.24$ whereas typical GRB afterglows show $\beta_{\mathrm{OX}} \sim 0.5-1.0$ (Gehrels et al. 2008). We also note that the decline of the light curve is better fit by exponential decay than by a power law (Section 3.4, Figure 8 (right)).

While the properties of the luminous blue peak of iPTF 16asu do not seem to resemble a classical GRB afterglow (on- or offaxis), it is worth noting that low-luminosity GRBs like 060218 and $100316 \mathrm{D}$ have shown thermal emission in addition to the weak synchrotron component (e.g., Campana et al. 2006; Starling et al. 2011). Thus it is still possible that iPTF 16asu could be a related phenomenon but with a significantly brighter thermal component. The origin of the thermal emission in lowluminosity GRBs is debated, though one possibility is that it is associated with shock breakout. We consider next whether such a model can also explain iPTF 16asu.

\subsection{Shock Cooling}

The short timescales and blue colors of iPTF 16asu are reminiscent of shock cooling transients, where the early light curve of an SN is powered by the cooling of the envelope following the breakout of the SN shock, usually followed by a second peak from the SN itself (e.g., SN 1993J; Wheeler et al. 1993). Such a shock cooling phase should be present in all SNe (Nakar \& Sari 2010), but both the duration and the luminosity will depend on the structure of the progenitor star. A peak in both the red and blue bands, as we see in iPTF 16asu, is generally associated with shock breakout from extended material (Nakar \& Piro 2014). Shock cooling models have been considered for other rapidly evolving transients (e.g.,
Ofek et al. 2010; Drout et al. 2014) as well as low-luminosity GRBs (e.g., Nakar 2015), so we consider here whether iPTF 16asu could be explained by a shock cooling scenario.

Since the peak is seen in all bands, we consider the extended envelope model of Nakar \& Piro (2014). Here the mass in the extended envelope scales as $M_{e} \propto \kappa^{-1} v t_{\text {peak }}^{2}$ and the effective radius of the material scales as $R_{e} \propto \kappa L_{\text {peak }} v^{-2}$. For the rise time and peak luminosity measured for iPTF 16asu, this suggests an envelope mass of around $\sim 0.5 M_{\odot}$ and a lower limit on the effective radius of the material of $\sim 2 \times 10^{12} \mathrm{~cm}$, still assuming $\kappa=0.1 \mathrm{~cm}^{2} \mathrm{~g}^{-1}$.

Piro (2015) developed this extended envelope further, and Figure 19 shows a fit of the model with the parameters $M_{\mathrm{ej}}=0.45 M_{\odot}, R_{\mathrm{e}}=1.7 \times 10^{12} \mathrm{~cm}$, and $E=3.8 \times 10^{51} \mathrm{erg}$. In general there is a degeneracy between the initial radius of the material and the energy deposited by the shock, but our high observed velocities suggest that we are in the regime of a smaller radius and higher energy (Piro 2015). Since the energy deposited into the extended material is just a fraction of the total SN energy, if this model is correct then it would imply a very high explosion energy, likely requiring a central engine.

Unlike many shock-breakout SNe, iPTF 16asu exhibits only a single peak, so if the main light curve peak is powered by shock cooling then it must completely dominate the contribution from the underlying normal SN light curve. The model shown in Figure 19 approximates the shock cooling light curve with a Gaussian and is not expected to capture the decline of the light curve, which would depend on the density structure of the material. It does, however, demonstrate that an extended envelope model can produce a peak with a rise time and luminosity compatible with iPTF 16asu. As seen in Figure 16, an SN Ic-BL slightly less luminous than SN 1998bw could be hidden underneath a luminous shock-breakout peak forming one continuous peak by the merging of the second SN peak with the decay of the first peak.

In Nakar (2015), SN 2006aj/GRB 060218 was modeled by a shock breakout from energy deposited into an extended (>100 $\left.R_{\odot}\right)$ low-mass $\left(\sim 0.01 M_{\odot}\right)$ envelope by a low-luminosity GRB. Thus iPTF 16asu could have a similar explosion mechanism but with a significantly higher-mass envelope, producing a longer-duration and luminous peak. The presence of circumstellar material would also be consistent with the constraints on high-energy emission; indeed, it has been suggested that low-energy soft GRBs like 060218 and 100316D have extended circumstellar material (Margutti et al. 2015).

\section{Summary}

We present photometric and spectroscopic observations of the unique transient iPTF 16asu. The key observed properties can be summarized as follows:

1. A rapidly evolving and luminous light curve, with a rise of 4.0 days to a high peak luminosity of $3.4 \times$ $10^{43} \mathrm{erg} \mathrm{s}^{-1}$. The decline is similarly fast and is well fit by exponential decay with a characteristic timescale of 14 days.

2. A blue and featureless spectrum near peak that is well fit by a blackbody, using UV and optical data, with a temperature of $\sim 11,000 \mathrm{~K}$ and a radius of $\sim 2.5 \times 10^{15} \mathrm{~cm}$.

3. Broad spectroscopic features emerging on the decline that are well matched to $\mathrm{SNe}$ Ic-BL. The velocities, as 


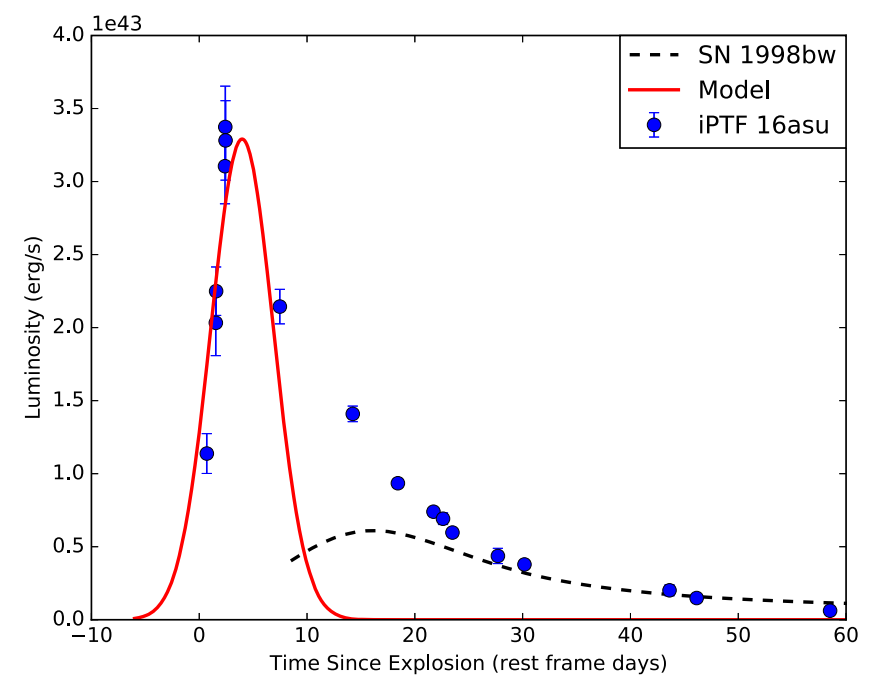

Figure 19. Fit of shock-breakout model (red) from Piro (2015) using the parameters $\kappa=0.1 \mathrm{~cm}^{2} \mathrm{~g}^{-1}, M_{\mathrm{e}}=0.45 M_{\odot} R_{\mathrm{e}}=1.7 \times 10^{12} \mathrm{~cm}\left(\sim 25 R_{\odot}\right)$, and $E_{e}=3.8 \times 10^{51} \mathrm{erg}$. The pseudobolometric light curve using $B V(R I)_{\mathrm{c}}$ bands of SN 1998bw (Clocchiatti et al. 2011) is plotted in black to illustrate how ${ }^{56} \mathrm{Ni}$ decay could power the late-time light curve.

measured from the Fe II $\lambda 5169 \AA$ line, are comparable to SNe Ic-BL with accompanying GRBs.

4. Nondetections in the X-ray (Swift/XRT) corresponding to limits of $(1-2) \times 10^{43} \mathrm{erg} \mathrm{s}^{-1}$ and in the radio (VLA) corresponding to limits of $(1-2) \times 10^{28} \mathrm{erg} \mathrm{s}^{-1}$. Nondetections by all-sky gamma-ray monitors similarly constrain any associated on-axis GRB to be lowenergy $\left(E_{\text {iso }}<10^{50} \mathrm{erg}\right)$.

5. A dwarf host galaxy, with a stellar mass of $\sim 5 \times 10^{8} M_{\odot}$, a metallicity of $Z \sim 0.3 Z_{\odot}$, and a star formation rate of $\sim 0.7 M_{\odot} \mathrm{yr}^{-1}$.

We discuss various energy sources to explain the above observed properties. We find that ${ }^{56} \mathrm{Ni}$ decay, as in an ordinary $\mathrm{SN}$ Ic-BL, is adequate to explain the late-time photometry. It is also consistent with the observed spectra and nondetections in the X-ray and radio bands. However, attempting to fit the rapid rise and luminous peak solely with ${ }^{56} \mathrm{Ni}$ decay gives the unphysical result that $M_{\mathrm{Ni}}>M_{\mathrm{ej}}$. Hence we consider two different hypotheses to explain the early data.

First we consider a magnetar model. The magnetar model either requires a very small ejecta mass $\left(0.086 M_{\odot}\right)$ in order to fit the sharp rise or a high magnetic field $\left(B=4.4 \times 10^{15} \mathrm{G}\right)$ that decreases the spin-down time. The latter would require that the late-time data are explained by the radioactive decay of ${ }^{56} \mathrm{Ni}$.

Next we find that shock cooling can also explain the fast rise and high luminosity with a dense envelope $\left(M_{\mathrm{e}}=0.45 M_{\odot}\right.$, $\left.R_{\mathrm{e}}=1.7 \times 10^{12} \mathrm{~cm}\right)$ and high injected energy $\left(E_{\mathrm{e}}=\right.$ $\left.3.8 \times 10^{51} \mathrm{erg}\right)$. The required energetics in this model also implies an underlying central engine. Shock cooling through the envelope has been seen in the low-luminosity SN 2006aj/ GRB 060218. Our spectra and kinematics are also more similar to $\mathrm{SNe}$ Ic-BL associated with GRBs. Our radio and X-ray limits constrain the energy $\left(E_{\text {iso }}\right)$ of any associated GRB to be $<10^{50} \mathrm{erg}$. Regardless of whether or not there was a GRB, the late-time light curve is reasonably fit by ${ }^{56} \mathrm{Ni}$ decay.

Both of the above scenarios suggest that iPTF 16asu was an engine-driven $\mathrm{SN}$, making it an intriguing transition object between SLSNe, low-luminosity GRBs, SNe Ic-BL, and objects like SN 2011kl. We hope that new discoveries from the next generation of wide-field surveys (e.g., Zwicky Transient Facility; Bellm \& Kulkarni 2017) will enable us to find more objects like iPTF 16asu and more conclusively determine the origins of such fast and luminous transients.

We thank the anonymous referee for constructive comments that improved the manuscript. We thank Iair Arcavi, Maria Drout, Avishay Gal-Yam, Raffaella Margutti, and Maryam Modjaz for helpful discussions and comments. R. L. acknowledges helpful discussions at the Munich Institute for Astro- and Particle Physics (MIAPP) workshop "Superluminous Supernovae in the Next Decade," supported by the MIAPP, of the DFG cluster of excellence "Origin and Structure of the Universe." We thank Harish Vedantham, Vikram Ravi, and Anna Ho for assisting with the observations presented in this paper. The Intermediate Palomar Transient Factory project is a scientific collaboration among the California Institute of Technology; Los Alamos National Laboratory; the University of Wisconsin, Milwaukee; the Oskar Klein Center; the Weizmann Institute of Science; the TANGO Program of the University System of Taiwan; and the Kavli Institute for the Physics and Mathematics of the Universe. This work was supported by the GROWTH project, funded by the National Science Foundation under Grant No. 1545949. This work is partially based on data acquired with the Swift GO program 1215281 (Grant No. NNX16AN84G, PI: R. Lunnan). Part of this research was carried out at the Jet Propulsion Laboratory, California Institute of Technology, under a contract with NASA. The National Radio Astronomy Observatory is a facility of the National Science Foundation operated under cooperative agreement by Associated Universities, Inc. A. C. acknowledges support from the National Science Foundation CAREER Award No. 1455090. D. S. and D. F. gratefully acknowledge support from RSF Grant No. 17-12-01378. This work made use of the data products generated by the New York University SN group and released under DOI:10.5281/zenodo.58767, available at https://github.com/ nyusngroup/SESNspectraLib based on observations made with the Nordic Optical Telescope, operated by the Nordic Optical Telescope Scientific Association at the Observatorio del Roque de los Muchachos, La Palma, Spain, of the Instituto de Astrofisica de Canarias. Some of the data presented herein were obtained at the W. M. Keck Observatory, which is operated as a scientific partnership among the California Institute of Technology, the University of California, and NASA. The Observatory was made possible by the generous financial support of the W. M. Keck Foundation. The authors wish to recognize and acknowledge the very significant cultural role and reverence that the summit of Mauna Kea has always had within the indigenous Hawaiian community. We are most fortunate to have the opportunity to conduct observations from this mountain.

Facilities: PO: 1.2 m, PO: 1.5 m, PO: Hale, Keck: I, Keck: II, NOT, TNG, Swift, VLA.

\section{ORCID iDs}

R. Lunnan (iD https://orcid.org/0000-0001-9454-4639

M. M. Kasliwal (i) https://orcid.org/0000-0002-5619-4938

D. A. Perley (iD https://orcid.org/0000-0001-8472-1996

A. Corsi (i) https://orcid.org/0000-0001-8104-3536

S. B. Cenko (i) https://orcid.org/0000-0003-1673-970X

N. Blagorodnova (iD https://orcid.org/0000-0003-0901-1606 
Y. Cao (ib https://orcid.org/0000-0002-8036-8491

D. O. Cook (10) https://orcid.org/0000-0002-6877-7655

G. B. Doran (1) https://orcid.org/0000-0003-1233-2224

D. D. Frederiks (10 https://orcid.org/0000-0002-1153-6340

C. Fremling (1) https://orcid.org/0000-0002-4223-103X

K. Hurley (i) https://orcid.org/0000-0003-3315-1975

E. Karamehmetoglu (i) https://orcid.org/0000-00016209-838X

S. R. Kulkarni (1) https://orcid.org/0000-0001-5390-8563

G. Leloudas (1) https://orcid.org/0000-0002-8597-0756

F. Masci (i) https://orcid.org/0000-0002-8532-9395

P. E. Nugent (1) https://orcid.org/0000-0002-3389-0586

A. Rubin (1) https://orcid.org/0000-0003-4557-0632

V. Savchenko (1) https://orcid.org/0000-0001-6353-0808

J. Sollerman (1) https://orcid.org/0000-0003-1546-6615

D. S. Svinkin 나 https://orcid.org/0000-0002-2208-2196

P. Vreeswijk (i) https://orcid.org/0000-0002-7572-9088

\section{References}

Aptekar, R. L., Frederiks, D. D., Golenetskii, S. V., et al. 1995, SSRv, 71, 265 Arcavi, I., Wolf, W. M., Howell, D. A., et al. 2016, ApJ, 819, 35

Arnett, W. D. 1982, ApJ, 253, 785

Asplund, M., Grevesse, N., Sauval, A. J., \& Scott, P. 2009, ARA\&A, 47, 481

Barbary, K., Dawson, K. S., Tokita, K., et al. 2009, ApJ, 690, 1358

Barnes, J., Duffell, P. C., Liu, Y., et al. 2017, arXiv:1708.02630

Bellm, E. C., \& Kulkarni, S. R. 2017, NatAs, 1, 0071

Bersten, M. C., Benvenuto, O. G., Orellana, M., \& Nomoto, K. 2016, ApJL, 817, L8

Bianco, F. B., Modjaz, M., Hicken, M., et al. 2014, ApJS, 213, 19

Bianco, F. B., Modjaz, M., Oh, S. M., et al. 2016, A\&C, 16, 54

Bildsten, L., Shen, K. J., Weinberg, N. N., \& Nelemans, G. 2007, ApJL, 662, L95

Brown, P. J., Breeveld, A. A., Holland, S., Kuin, P., \& Pritchard, T. 2014, Ap\&SS, 354, 89

Burrows, D. N., Hill, J. E., Nousek, J. A., et al. 2005, SSRv, 120, 165

Campana, S., Mangano, V., Blustin, A. J., et al. 2006, Natur, 442, 1008

Cao, Y., Nugent, P. E., \& Kasliwal, M. M. 2016, PASP, 128, 114502

Cardelli, J. A., Clayton, G. C., \& Mathis, J. S. 1989, ApJ, 345, 245

Cenko, S. B., Fox, D. B., Moon, D.-S., et al. 2006, PASP, 118, 1396

Chandra, P., \& Frail, D. A. 2012, ApJ, 746, 156

Chomiuk, L., Chornock, R., Soderberg, A. M., et al. 2011, ApJ, 743, 114

Clocchiatti, A., Suntzeff, N. B., Covarrubias, R., \& Candia, P. 2011, AJ, 141,163

Corsi, A., Cenko, S. B., Kasliwal, M. M., et al. 2017, ApJ, 847, 57

Corsi, A., Ofek, E. O., Gal-Yam, A., et al. 2012, ApJL, 747, L5

Dessart, L., Hillier, D. J., Yoon, S.-C., Waldman, R., \& Livne, E. 2017, A\&A, 603, A51

Drout, M. R., Chornock, R., Soderberg, A. M., et al. 2014, ApJ, 794, 23

Evans, P. A., Beardmore, A. P., Page, K. L., et al. 2007, A\&A, 469, 379

Faber, S. M., Phillips, A. C., Kibrick, R. I., et al. 2003, Proc. SPIE, 4841, 1657 Filippenko, A. V. 1997, ARA\&A, 35, 309

Foley, R. J., Papenkova, M. S., Swift, B. J., et al. 2003, PASP, 115, 1220

Fremling, C., Sollerman, J., Taddia, F., et al. 2016, A\&A, 593, A68

Galama, T. J., Groot, P. J., van Paradijs, J., et al. 1998a, ApJL, 497, L13

Galama, T. J., Vreeswijk, P. M., van Paradijs, J., et al. 1998b, Natur, 395, 670 Gal-Yam, A. 2012, Sci, 337, 927

Gehrels, N., Barthelmy, S. D., Burrows, D. N., et al. 2008, ApJ, 689, 1161

Greiner, J., Mazzali, P. A., Kann, D. A., et al. 2015, Natur, 523, 189

Hayden, B. T., Garnavich, P. M., Kessler, R., et al. 2010, ApJ, 712, 350

Högbom, J. A. 1974, A\&AS, 15, 417

Hogg, D. W., Baldry, I. K., Blanton, M. R., \& Eisenstein, D. J. 2002, arXiv: astro-ph/0210394

Hosseinzadeh, G., Arcavi, I., Valenti, S., et al. 2017, ApJ, 836, 158

Inserra, C., Smartt, S. J., Jerkstrand, A., et al. 2013, ApJ, 770, 128

Jones, E., Oliphant, T., Peterson, P., et al. 2001, SciPy: Open Source Scientific Tools for Python

Kangas, T., Blagorodnova, N., Mattila, S., et al. 2017, MNRAS, 469, 1246

Kann, D. A., Klose, S., Zhang, B., et al. 2010, ApJ, 720, 1513

Kann, D. A., Schady, P., Olivares, E., et al. 2016, A\&A, submitted (arXiv:1606.06791)

Kasen, D., \& Bildsten, L. 2010, ApJ, 717, 245
Kasliwal, M. M. 2012, PASA, 29, 482

Kasliwal, M. M., Kulkarni, S. R., Gal-Yam, A., et al. 2010, ApJL, 723, L98

Kasliwal, M. M., Kulkarni, S. R., Gal-Yam, A., et al. 2012, ApJ, 755, 161

Kennicutt, R. C. , Jr. 1998, ARA\&A, 36, 189

Kewley, L. J., \& Dopita, M. A. 2002, ApJS, 142, 35

Kewley, L. J., \& Ellison, S. L. 2008, ApJ, 681, 1183

Kobulnicky, H. A., \& Kewley, L. J. 2004, ApJ, 617, 240

Kriek, M., van Dokkum, P. G., Labbé, I., et al. 2009, ApJ, 700, 221

Law, N. M., Kulkarni, S. R., Dekany, R. G., et al. 2009, PASP, 121, 1395

Leloudas, G., Schulze, S., Krühler, T., et al. 2015, MNRAS, 449, 917

Levesque, E. M., Berger, E., Kewley, L. J., \& Bagley, M. M. 2010, AJ, 139,694

Liu, Y.-Q., Modjaz, M., Bianco, F. B., \& Graur, O. 2016, ApJ, 827, 90

Lunnan, R., Chornock, R., Berger, E., et al. 2013, ApJ, 771, 97

Lunnan, R., Chornock, R., Berger, E., et al. 2014, ApJ, 787, 138

Lunnan, R., Kasliwal, M. M., Cao, Y., et al. 2017, ApJ, 836, 60

Lyman, J. D., Bersier, D., \& James, P. A. 2014, MNRAS, 437, 3848

Lyman, J. D., Bersier, D., James, P. A., et al. 2016, MNRAS, 457, 328

Maraston, C. 2005, MNRAS, 362, 799

Margutti, R., Guidorzi, C., Lazzati, D., et al. 2015, ApJ, 805, 159

Masci, F. J., Laher, R. R., Rebbapragada, U. D., et al. 2017, PASP, 129, 014002

Matheson, T., Filippenko, A. V., Ho, L. C., Barth, A. J., \& Leonard, D. C. 2000, AJ, 120, 1499

McGaugh, S. S. 1991, ApJ, 380, 140

McKenzie, E. H., \& Schaefer, B. E. 1999, PASP, 111, 964

McMullin, J. P., Waters, B., Schiebel, D., Young, W., \& Golap, K. 2007, in ASP Conf. Ser. 376, Astronomical Data Analysis Software and Systems XVI, ed. R. A. Shaw, F. Hill, \& D. J. Bell (San Francisco, CA: ASP), 127

Metzger, B. D., Margalit, B., Kasen, D., \& Quataert, E. 2015, MNRAS, 454, 3311

Modjaz, M., Liu, Y. Q., Bianco, F. B., \& Graur, O. 2016, ApJ, 832, 108

Modjaz, M., Stanek, K. Z., Garnavich, P. M., et al. 2006, ApJL, 645, L21

Nakar, E. 2015, ApJ, 807, 172

Nakar, E., \& Piro, A. L. 2014, ApJ, 788, 193

Nakar, E., \& Sari, R. 2010, ApJ, 725, 904

Ofek, E. O., Rabinak, I., Neill, J. D., et al. 2010, ApJ, 724, 1396

Oke, J. B., Cohen, J. G., Carr, M., et al. 1995, PASP, 107, 375

Oke, J. B., \& Gunn, J. E. 1982, PASP, 94, 586

Osterbrock, D. E. 1989, Astrophysics of Gaseous Nebulae and Active Galactic Nuclei (Mill Valley, CA: Univ. Science Books)

Pastorello, A., Smartt, S. J., Botticella, M. T., et al. 2010, ApJL, 724, L16

Patat, F., Cappellaro, E., Danziger, J., et al. 2001, ApJ, 555, 900

Perets, H. B., Gal-Yam, A., Mazzali, P. A., et al. 2010, Natur, 465, 322

Perley, D. A., Cenko, S. B., Corsi, A., et al. 2014, ApJ, 781, 37

Perley, D. A., Quimby, R. M., Yan, L., et al. 2016, ApJ, 830, 13

Pettini, M., \& Pagel, B. E. J. 2004, MNRAS, 348, L59

Phillips, M. M. 1993, ApJL, 413, L105

Piro, A. L. 2015, ApJL, 808, L51

Prieto, J. L., Kistler, M. D., Thompson, T. A., et al. 2008, ApJL, 681, L9

Quimby, R. M., Kulkarni, S. R., Kasliwal, M. M., et al. 2011, Natur, 474, 487

Roming, P. W. A., Kennedy, T. E., Mason, K. O., et al. 2005, SSRv, 120, 95

Rubin, A., Gal-Yam, A., De Cia, A., et al. 2016, ApJ, 820, 33

Sanders, N. E., Soderberg, A. M., Levesque, E. M., et al. 2012, ApJ, 758, 132

Sari, R., Piran, T., \& Narayan, R. 1998, ApJL, 497, L17

Schlafly, E. F., \& Finkbeiner, D. P. 2011, ApJ, 737, 103

SDSS Collaboration, Albareti, F. D., Allende Prieto, C., et al. 2016, ApJS, in press (arXiv:1608.02013)

Shen, K. J., Kasen, D., Weinberg, N. N., Bildsten, L., \& Scannapieco, E. 2010, ApJ, 715, 767

Soderberg, A. M., Chakraborti, S., Pignata, G., et al. 2010, Natur, 463, 513

Starling, R. L. C., Wiersema, K., Levan, A. J., et al. 2011, MNRAS, 411, 2792

Taddia, F., Sollerman, J., Leloudas, G., et al. 2015, A\&A, 574, A60

Tiengo, A., Mereghetti, S., Ghisellini, G., Tavecchio, F., \& Ghirlanda, G. 2004 A\&A, 423, 861

van Eerten, H., Zhang, W., \& MacFadyen, A. 2010, ApJ, 722, 235

van Eerten, H. J., \& MacFadyen, A. I. 2011, ApJL, 733, L37

von Kienlin, A., Beckmann, V., Rau, A., et al. 2003, A\&A, 411, L299

Wheeler, J. C., Barker, E., Benjamin, R., et al. 1993, ApJL, 417, L71

Wheeler, J. C., Johnson, V., \& Clocchiatti, A. 2015, MNRAS, 450, 1295

Woosley, S. E. 2010, ApJL, 719, L204

Woosley, S. E., \& Bloom, J. S. 2006, ARA\&A, 44, 507

Woosley, S. E., Eastman, R. G., Weaver, T. A., \& Pinto, P. A. 1994, ApJ, 429, 300

Yaron, O., \& Gal-Yam, A. 2012, PASP, 124, 668 